\title{
Magnetic fields and Faraday rotation in clusters of galaxies
}

\author{
M. Murgia ${ }^{1,2}$, F. Govoni ${ }^{1,3}$, L. Feretti ${ }^{1}$, G. Giovannini ${ }^{1,3}$, D. Dallacasa ${ }^{1,3}$, R. Fanti ${ }^{1,4}$, G. B. Taylor ${ }^{5}$, and K. Dolag ${ }^{6}$ \\ ${ }^{1}$ Istituto di Radioastronomia del CNR, via Gobetti 101, 40129 Bologna, Italy \\ e-mail: murgia@ira.cnr.it \\ 2 INAF - Osservatorio Astronomico di Cagliari, Loc. Poggio dei Pini, Strada 54, 09012 Capoterra (CA), Italy \\ 3 Dipartimento di Astronomia, Università di Bologna, via Ranzani 1, 40127 Bologna, Italy \\ 4 Dipartimento di Fisica, Università di Bologna, via Irnerio 46, 40126 Bologna, Italy \\ 5 National Radio Astronomy Observatory, Socorro, NM 87801, USA \\ ${ }^{6}$ Dipartimento di Astronomia, Università di Padova, vicolo dell'Osservatorio 5, 35122 Padova, Italy
}

Received 7 July 2003 / Accepted 3 June 2004

\begin{abstract}
We present a numerical approach to investigate the relationship between magnetic fields and Faraday rotation effects in clusters of galaxies. We can infer the structure and strength of intra-cluster magnetic fields by comparing our simulations with the observed polarization properties of extended cluster radio sources such as radio galaxies and halos. We find the observations require a magnetic field which fluctuates over a wide range of spatial scales (at least one order of magnitude). If several polarized radio sources are located at different projected positions in a galaxy cluster, as is the case for A119, detailed Faraday rotation images allow us to constrain both the magnetic field strength and the slope of the power spectrum. Our results show that the standard analytic expressions applied in the literature overestimate the cluster magnetic field strengths by a factor of $\sim 2$. We investigate the possible effects of our models on beam depolarization of radio sources whose radiation traverses the magnetized intracluster medium. Finally, we point out that radio halos may provide important information about the spatial power spectrum of the magnetic field fluctuations on large scales. In particular, different values of the index of the power spectrum produce very different total intensity and polarization brightness distributions.
\end{abstract}

Key words. magnetic fields - galaxies: clusters: general

\section{Introduction}

The existence of cluster scale magnetic fields has been demonstrated in different ways. Some clusters of galaxies exhibit non-thermal synchrotron radio halos which extend up to Megaparsec scales. These diffuse radio sources constitute direct evidence for large scale magnetic fields associated with the intracluster medium. Indirect evidence for cluster scale magnetic fields are provided by the observation of Faraday rotation in radio sources located inside or behind galaxy clusters. For a review of these and other techniques see Carilli \& Taylor (2002).

A crucial issue concerns the effective strength and structure of cluster magnetic fields since different methods of analysis give somewhat discrepant estimates for the field strengths. Due to their low surface brightness, radio halos have been studied so far with low spatial resolution. This prevents a detailed observation of the small scale magnetic field geometry and intensity. However, using minimum energy assumptions, it is possible to estimate an equipartition magnetic field strength averaged over the entire halo volume. These estimates give equipartition magnetic field strengths of $\simeq 0.1$ to $1 \mu \mathrm{G}$ (e.g., Feretti \& Giovannini 1996; Govoni et al. 2001a; Bacchi et al. 2003).
In a few cases, clusters containing a radio halo show a hard $\mathrm{X}$-ray excess emission. This emission could be interpreted in terms of Inverse Compton scattering of the cosmic microwave background photons with the relativistic electrons responsible for the radio halo emission. In this case, the measures of the magnetic field strength (e.g., Fusco-Femiano et al. 1999; Rephaeli et al. 1999, Rephaeli \& Gruber 2002; Fusco-Femiano et al. 2004) inferred from the ratio of the radio to X-ray luminosities are consistent with the equipartition estimates. Because of the lack of spatial information of these X-ray observations also in this case we obtain a field strength averaged over a large region. Indirect measurements of the magnetic field strength can also be determined in conjunction with X-ray observations of the hot gas, through the study of the Faraday Rotation Measure (RM) of radio sources located inside or behind clusters. By using simple analytical approaches, relatively strong magnetic fields, from $\sim 5 \mu \mathrm{G}$ up to the values of $30 \mu \mathrm{G}$ have been found in cooling flow clusters (e.g., 3C 295, Perley \& Taylor 1991; Allen et al. 2001; Hydra A, Taylor \& Perley 1993) where extremely high RMs have been revealed. On the other hand, significant magnetic fields have also been detected in clusters without cooling flows: the RM measurements of 
polarized radio sources through the hot intracluster medium leads to a magnetic field of $2-8 \mu \mathrm{G}$ with patchy structures on small scales in the range $2-15 \mathrm{kpc}$ (e.g., Feretti et al. 1995, 1999a; Govoni et al. 2001b; Taylor et al. 2001; Eilek \& Owen 2002). From a statistical study of several clusters, Clarke et al. (2001) obtained magnetic fields of $4-8 \mu \mathrm{G}$, in agreement with the above findings.

The magnetic field strength obtained by RM studies is therefore higher than the value derived from the radio halo data and from Inverse-Compton X-ray studies. However, as pointed out by Carilli \& Taylor (2002, and references therein), all the aforementioned techniques are based on several assumptions. For example, the observed RMs have been interpreted until now in terms of simple analytical models which consider single-scale magnetic fields, while equipartition calculations in radio halos assume spatially uniform magnetic fields.

Newman et al. (2002) demonstrated that the assumption of a single-scale magnetic field leads to an overestimation of the magnetic field strength calculated through RM studies. Moreover, detailed magneto-hydrodynamic cosmological simulations (Dolag et al. 2002) suggested that the cluster magnetic fields may span a wide range of spatial scales with a strength that decreases with distance from the cluster center.

Finally, It has been pointed out that in some cases a radio source could compress the gas and fields in the ICM to produce local RM enhancements, thus leading to overestimates of the derived ICM magnetic field strength (e.g., Feretti et al. 1995; Rudnick \& Blundell 2003). No investigation in this respect has been attempted in this work.

So far, very little attention has been given in the literature to the determination of the power spectrum of the intracluster magnetic field fluctuations. Only recently Enßlin \& Vogt (2003) and Vogt \& Enßlin (2003) by using a new semianalytic technique showed that, for those cluster sources for which a very detailed RM image is available, the magnetic field power spectrum can be estimated.

The aim of our work is to provide an alternative numerical approach for investigating the strength and structure of cluster magnetic fields through Monte Carlo simulations. The simulations results are compared to the data through an interactive technique. The algorithms are assembled into a software package named FARADAY.

In particular, we will use FARADAY to study the power spectrum of the intra-cluster magnetic field from two different but complementary points of view. First, we show that both the mean and the dispersion of the RM can be used (and therefore should be used) to constrain not only the strength but also the power spectrum slope of the magnetic field fluctuations. In a consistent way, our simulations can be also employed to derive the expected de-polarization of cluster sources, such as radio galaxies and large scale halos, thus improving the estimates based on the RM analysis.

Throughout this paper we will assume a simple power law form for the magnetic field power spectrum and compare the observed cluster radio source polarization and RM images with the simulated values over regions of comparable size. The reader should be aware that many results presented in this work are influenced by the form assumed for the power spectrum and by the limitations of the numerical simulations. However, by considering the magnetic field strength as radial dependent and by modeling the magnetic field fluctuations within a multiscale length approach we made a new step towards more realistic cluster magnetic fields models. As we will show, modeling of magnetic fields as those we propose are necessary to interpret Faraday rotation effects in galaxy clusters and should be seen as a starting point for a new line of investigations.

This paper is organized as follows: in Sect. 2 we give a brief description of the FARADAY tool. In Sect. 3 we discuss the three-dimensional multi-scale cluster magnetic fields models as implemented in the program and used in our simulations. In Sect. 4 we simulate the Rotation Measure. In Sect. 5 we simulate the beam depolarization of cluster radio galaxies and the morphology and depolarization of the radio halo emission. In Sect. 6 we present some comparisons of simulations with real data. Some discussion and conclusions are presented in Sect. 7.

In this work we adopt a Hubble constant $H_{0}=$ $50 \mathrm{~km} \mathrm{~s}^{-1} \mathrm{Mpc}^{-1}$ and a deceleration parameter $q_{0}=0.5$.

\section{Faraday rotation in clusters of galaxies and the FARADAY tool}

The polarized synchrotron radiation incoming from radio sources located inside or behind a galaxy cluster, experiences a rotation of the plane of polarization as it passes through the magnetized and ionized intra-cluster medium:

$\Psi_{\mathrm{Obs}}(v)=\Psi_{\mathrm{Int}}+(c / v)^{2} \times \mathrm{RM}$

where $\Psi_{\mathrm{Obs}}(v)$ is the observed polarization angle at a frequency $v$ and $\Psi_{\text {Int }}$ is the intrinsic polarization angle.

The RM is related to the thermal electron density, $n_{\mathrm{e}}$, and magnetic field along the line-of-sight, $B_{\|}$, through the cluster by the equation:

$\mathrm{RM}=812 \int_{0}^{L} n_{\mathrm{e}} B_{\|} \mathrm{d} l \mathrm{rad} \mathrm{m}^{-2}$

where $B_{\|}$is measured in $\mu \mathrm{G}, n_{\mathrm{e}}$ in $\mathrm{cm}^{-3}$ and $L$ is the depth of the screen in kpc.

The position angle of the polarization plane is an observable quantity, therefore, RM images of radio sources can be constructed, by a linear fit to Eq. (1). The observed degree of polarization intensity, $P_{\mathrm{Obs}}(v)$, can be significantly lower with respect to the intrinsic value, $P_{\text {Int }}$, if differential Faraday rotation occurs within the observing beam. The observable data, RM and (de)polarization, together with a model for the density distribution of the X-ray emitting hot gas can provide, in principle, important information on the cluster magnetic field strength and structure. However, there are a number of practical difficulties that complicate the applicability of this method. In particular, due to the random character of the cluster magnetic fields, Eq. (2) is not analytically solvable even for very simple distributions of the thermal electrons. Moreover, we will always observe a projection of the random field onto the twodimensional sky, so we would need to de-project the power 
spectrum and the auto-correlation function radio source RM images in order to reconstruct the magnetic field configuration. This may be a non-trivial process (Enßlin \& Vogt 2003).

For this purpose we developed the simulation tool FARADAY which allows an interactive, numerical approach for investigating cluster magnetic fields.

The thermal electron gas density of the screen is generally not known, and we then assume a standard $\beta$-model distribution:

$n_{\mathrm{e}}(r)=n_{0}\left(1+r^{2} / r_{\mathrm{c}}^{2}\right)^{-3 \beta / 2}$

where $r, n_{0}$ and $r_{\mathrm{c}}$ are the distance from the cluster center, the central electron density, and the cluster core radius, respectively.

By following some simple steps it is possible to compute the effects of a cluster magnetic field model on the polarization properties of sources located inside or behind a cluster. Given a three-dimensional magnetic field model and the density distribution of the intra-cluster gas, FARADAY calculates the RM image by integrating Eq. (2) numerically.

The beam depolarization is expected to be particularly strong if the radio source is observed with a beam larger than the minimum magnetic field scale length. This effect can be studied with FARADAY as well. Starting from the intrinsic polarization images of $P_{\text {Int }}$ and $\Psi_{\text {Int }}$, FARADAY can simulate the expected beam depolarization as a function of wavelength. One of the key feature of FARADAY is that it allows us to study the effect of magnetic field models that cannot be investigated analytically. In this work we use FARADAY to investigate the effects of a turbulent and isotropic magnetic fields which fluctuate over a range of spatial scales.

\section{A multi-scale magnetic field model}

Detailed images of the polarized emission are now available for a significant number of radio sources seen in galaxy clusters and indicate that the RM fluctuates down to linear scales as low as $10 \mathrm{kpc}$ or less (e.g., Carilli \& Taylor 2002, and references therein). If interpreted in terms of an external Faraday screen, the dispersion, $\sigma_{\mathrm{RM}}$, and mean, $\langle\mathrm{RM}\rangle$, of the RM fluctuations can be used to constrain the cluster magnetic field strength.

The effect of Faraday rotation from a tangled magnetic field has been analyzed by several authors (e.g., Lawler \& Dennison 1982; Tribble 1991a; Feretti et al. 1995; Felten 1996). In the simplest ideal case, the screen is made of cells of uniform size, electron density and magnetic field strength, but with a field orientation at random angles in each cell. The observed RM along any given line of sight is then generated by a random walk process involving a large number of cells of size $\Lambda_{c}$. The distribution of the RM is Gaussian with zero mean, and a variance given by:

$\sigma_{\mathrm{RM}}^{2}=\left\langle\mathrm{RM}^{2}\right\rangle=812^{2} \Lambda_{\mathrm{c}} \int\left(n_{\mathrm{e}} B_{\|}\right)^{2} \mathrm{~d} l$.

As we will show in Sect. 4.4, since the density profile of the ionized gas can be obtained by X-ray observations, the intergalactic magnetic field strength can be estimated by measuring $\sigma_{\mathrm{RM}}$ from spatially resolved RM images of radio sources in the cluster if $\Lambda_{\mathrm{c}}$ is known.
In many cases the observed RM distributions are nearly Gaussians, suggesting an isotropic distribution of the field component along the line-of-sight. However, many RM distributions show clear evidence for a non-zero $\langle\mathrm{RM}\rangle$ if averaged over areas comparable with the radio source size which is typically $50 \times 50 \mathrm{kpc}^{2}$. This same property is observed for clusters located at high galactic latitudes and in general cannot be entirely attributed to the contribution from our own Milky Way Galaxy. These $\langle\mathrm{RM}\rangle$ offsets are more likely due to fluctuations of the cluster magnetic fields on scales greater than $50-100 \mathrm{kpc}$, i.e. considerably larger of those responsible for the RM dispersion. The random magnetic field must therefore be both tangled on sufficiently small scales in order to produce the smallest structures observed in the RM images and also fluctuate on scales one, or even two, orders of magnitude larger. For this reason, it is necessary to consider cluster magnetic field models where both small and large scales structures coexist. To accomplish this we simulated in a cubical box a three-dimensional multiscale magnetic field.

Following the approach proposed by Tribble (1991b), the field is constructed by selecting a power spectrum for the vector potential $\boldsymbol{A}$ and choosing the Fourier components $\tilde{A}(\boldsymbol{k})$ accordingly.

For all grid points in the wave-numbers space the amplitude and phase, respectively $A$ and $\phi$, of each component of $\tilde{A}(\boldsymbol{k})$ are randomly drawn from the distribution:

$P(A, \phi) \mathrm{d} A \mathrm{~d} \phi=\frac{A}{2 \pi\left|A_{k}\right|^{2}} \exp \left(-\frac{A^{2}}{2\left|A_{k}\right|^{2}}\right) \mathrm{d} A \mathrm{~d} \phi$

i.e. $A$ is extracted from a Rayleigh distribution and $\phi$ is uniformly distributed between 0 and $2 \pi$.

We adopted a power-law power spectrum $^{1},\left|A_{k}\right|^{2}$, of the form:

$\left|A_{k}\right|^{2} \propto k^{-\zeta}$

The magnetic field:

$\tilde{B}(\boldsymbol{k})=i \boldsymbol{k} \times \tilde{A}(\boldsymbol{k})$

is then transformed back into the real space using a threedimensional Fast Fourier Transform (Press et al. 1986). This automatically ensures a tangled, divergence free, multi-scale magnetic field model whose components have a power spectrum which follows:

$\left|B_{k}\right|^{2}=C_{n}^{2} k^{-n}$

where $n=\zeta-2$ while $C_{n}^{2}$ is the power spectrum normalization.

The power spectrum (8) represents the magnetic field energy $\left(\mathrm{erg} \mathrm{cm}^{3}\right)$ associated with each wave-number.

The assumed form for the vector potential results in a magnetic field whose components, $B_{i}$, have a Gaussian distribution

\footnotetext{
1 Note that throughout this paper the power spectra are expressed as vectorial forms in $k$-space. The one-dimensional forms can be obtained by multiplying by $4 \pi k^{2}$ and $2 \pi k$ respectively the three and twodimensional power spectra.
} 
with $\left\langle B_{i}\right\rangle=0$ and $\sigma_{B_{i}}^{2}=\left\langle B_{i}^{2}\right\rangle$. The distribution of $B$ itself is therefore a Maxwellian with $\langle B\rangle=2 \sqrt{\frac{2}{\pi}} \sigma_{B_{i}}$ and $\sigma_{B}=$ $\sqrt{\frac{3 \pi-8}{\pi}} \sigma_{B_{i}}$.

From the Parseval's Theorem, $\int\left|B_{i}\right|^{2} \mathrm{~d} V=\int\left|B_{k}\right|^{2} \mathrm{~d}^{3} k$, it follows that the magnetic field energy density averaged over the volume, $V$, is proportional to

$\langle B\rangle^{2} \propto \frac{C_{n}^{2}}{V} \cdot \ln \left(k_{\max } / k_{\min }\right)$

for $n=3$, and

$\langle B\rangle^{2} \propto \frac{C_{n}^{2}}{V} \cdot \frac{k_{\max }^{3-n}-k_{\min }^{3-n}}{3-n}$

for $n \neq 3$. Where the wave-numbers $k_{\min }$ and $k_{\max }$ correspond, respectively, to the minimum and maximum spatial scale of the magnetic field.

\subsection{A magnetic field strength with a radial decrease}

Important clues about the radial gradient of the magnetic field strength in clusters of galaxies are provided by observations of clusters hosting a radio halo and by magneto-hydrodynamic cosmological simulations. The spatial correlation found in some clusters between the X-ray and the radio halo brightness (Govoni et al. 2001c) suggests that thermal and nonthermal (relativistic particles and magnetic fields) energy densities could have the same radial scaling. In the radio halo of the Coma cluster (Giovannini et al. 1993) a steepening of the synchrotron spectrum is observed from the cluster center outward. Such a spectral behavior is expected in some halo formation models which consider a radial decrease of the cluster magnetic field strength (e.g., Brunetti et al. 2002). Moreover, detailed magneto-hydrodynamic cosmological simulations (Dolag et al. 2002) lead to magnetic fields whose strength decreases with the distance from the cluster center.

Therefore in this work we considered a magnetic field whose strength decreases from the cluster center according to:

$$
\langle\boldsymbol{B}\rangle(r)=\langle\boldsymbol{B}\rangle_{0} \cdot\left(1+r^{2} / r_{\mathrm{c}}^{2}\right)^{-\frac{3}{2} \mu}
$$

where $\langle\boldsymbol{B}\rangle_{0}$ is the mean magnetic field at the cluster center. Given the electron density profile in Eq. (3), we have $\langle\boldsymbol{B}\rangle \propto$ $n_{\mathrm{e}}^{\mu / \beta}$. Therefore the case $\mu=\beta / 2$ corresponds to a magnetic field whose energy density decreases from the cluster center as the gas energy density while $\mu=2 \beta / 3$ corresponds to a magnetic field frozen in the matter.

By combining RM and X-ray data it is possible to obtain an estimate of the index $\mu$ (Dolag et al. 2001). The X-ray surface brightness, $S_{\mathrm{x}}$, in a case of the $\beta$-model (Eq. (3)), is proportional to

$S_{\mathrm{x}} \propto\left(1+r_{\perp}^{2} / r_{\mathrm{c}}^{2}\right)^{-3 \beta+\frac{1}{2}}$

while, by substituting Eqs. (11) in (4), the RM is proportional to $\left\langle\mathrm{RM}^{2}\right\rangle^{1 / 2} \propto\left(1+r_{\perp}^{2} / r_{\mathrm{c}}^{2}\right)^{-\frac{3}{2}(\beta+\mu)+\frac{1}{4}}$
Table 1. Parameters adopted in the simulations.

\begin{tabular}{ll}
\hline \hline Grid size & $512^{3}$ pixels \\
Cellsize & 1 pixel $=3 \mathrm{kpc}$ \\
\hline Core radius & $r_{\mathrm{c}}=400 \mathrm{kpc}$ \\
Central density & $n_{0}=10^{-3} \mathrm{~cm}^{-3}$ \\
Beta & $\beta=0.6$ \\
\hline Central Mean magnetic field & $\langle\boldsymbol{B}\rangle_{0}=1 \mu \mathrm{G}$ \\
Radial profile slope & $\mu=0,0.3$ \\
Magnetic field minimum scale & $\Lambda_{\min }=6 \mathrm{kpc}$ \\
Magnetic field maximum scale & $\Lambda_{\max }=768 \mathrm{kpc}$ \\
Power spectrum spectral index & $n=2,3,4$ \\
\hline
\end{tabular}

where $r_{\perp}$ is the projected distance from the cluster center. By comparing the two line-of-sight integrals, one finds that the in$\operatorname{dex} \mu$ is related to the slope, $\alpha$, of the correlation $\left\langle\mathrm{RM}^{2}\right\rangle^{1 / 2} \propto$ $S_{\mathrm{x}}^{\alpha}$ and to the density index, $\beta$, through

$\mu=(2 \alpha-1) \cdot(\beta-1 / 6)$.

Thus, for a constant magnetic field $(\mu=0)$ the slope of the $\mathrm{RM}-S_{\mathrm{x}}$ correlation should be $\alpha=0.5$ while a steeper slope would imply $\mu>0$.

Formally, a decreasing magnetic field strength, as in Eq. (11), should be achieved by convolving the vector potential components with the shaping profile in the Fourier space. A more practical, although less rigorous, way to obtain this, consists in shaping the magnetic field to the desired profile directly in real space. We checked that in our cases the two approaches give minimal differences and for simplicity we adopted the latter. It should be noted that the magnetic field power spectrum in both cases is given by the convolution of the power-law in Eq. (8) with the Fourier transform of the shaping function. However, since the core radius of the shaping function is a considerable fraction of the simulated volume the convolution does not significantly alter the form of the power-law in Eq. (8).

\section{Simulated rotation measures}

Using the cluster magnetic field model described in Sect. 3, we studied the behavior of the RM for different values of the spectral index of the power spectrum in the range from $n=2$ to $n=4$. The magnetic energy density scales as $\mathrm{d} u_{\mathrm{B}} / \mathrm{d} k \propto k^{2}\left|B_{k}\right|^{2}$, thus $n=3$ implies that the field is scale-invariant, i.e. the energy density per logarithmic wavenumber interval is constant. It follows that for $n<3$ the magnetic energy density is larger on the smaller scales while for $n>3$ most of the magnetic energy density is on the larger scales. We now explore all these possibilities.

\subsection{Simulated RM images}

We simulated the expected RM images corresponding to the three different values of the power spectrum spectral index mentioned above in the case of a cluster with typical size and gas density parameters. The computational grid, gas density and magnetic field model parameters used for these specific 

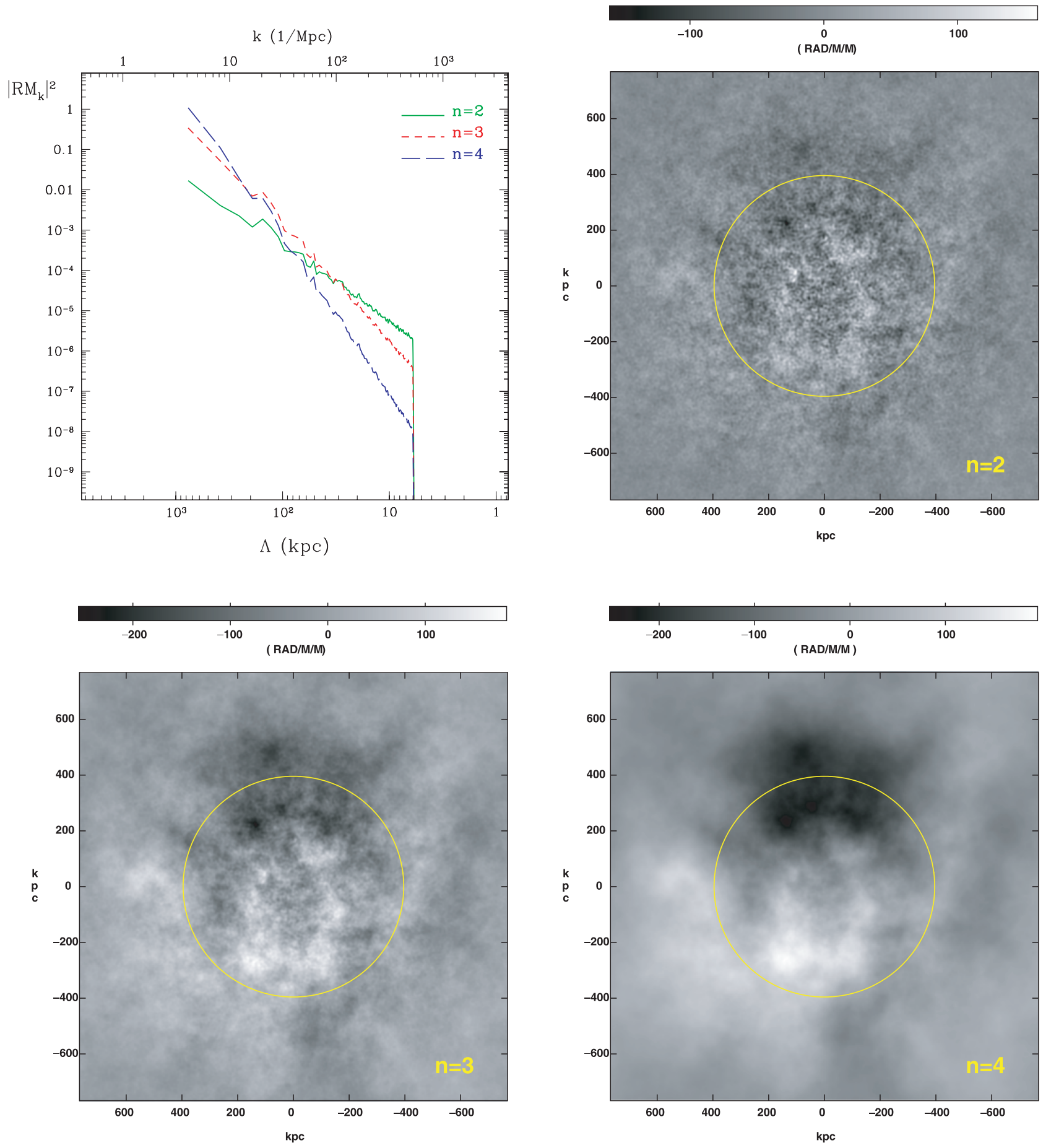

Fig. 1. Simulated RM images for different values of the magnetic field power spectrum spectral index $n$. The three power spectra are normalized to have the same total magnetic field energy which is distributed over the range of spatial scales from $6 \mathrm{kpc}$ up to $770 \mathrm{kpc}$. The average field at the cluster center is $\langle\boldsymbol{B}\rangle_{0}=1 \mu \mathrm{G}$ and its energy density decreases from the cluster center according to $B^{2} \propto n_{\mathrm{e}}(r)$, where $n_{\mathrm{e}}(r)$ is the gas density profile. Each RM image shows a field of view of about $1.5 \times 1.5 \mathrm{Mpc}$ while the cluster core radius (indicated by the circle) is $400 \mathrm{kpc}$. We performed the RM integration from the cluster center up to 3.8 core radii along the line-of-sight. The two-dimensional power spectra of the simulated RM images are shown in the top left panel. They have the same slope as their parent magnetic field power spectra and they span an equivalent range of spatial scales - see text for details.

simulations are listed in Table 1 while the RM images we obtained are shown in Fig. 1. Each RM image shows a field of view of about $1.5 \times 1.5 \mathrm{Mpc}$ while the cluster core radius (indicated by the circles in Fig. 1) is taken to be $400 \mathrm{kpc}$. We performed the integration of Eq. (2) from the cluster center up to $1.5 \mathrm{Mpc}$ (i.e. $3.8 r_{\mathrm{c}}$ ) along the line-of-sight. To do this we located the cluster center in the farthest side of the computational grid. The three magnetic field power spectra are normalized to generate the same average magnetic field energy density. The average field at the cluster center is $\langle\boldsymbol{B}\rangle_{0}=1 \mu \mathrm{G}$ and its energy 
density decreases from the cluster center as the gas energy density profile, i.e. $\mu=0.3$ (see Eq. (11)). This corresponds to a mean magnetic field of $\langle\boldsymbol{B}\rangle \simeq 0.46 \mu \mathrm{G}$ within a spherical volume with a radius of $r=3 r_{\mathrm{c}}$.

Since the size of the computational grid is finite only a limited range of spatial scales can be investigated. The grid is large enough to contain a cluster of typical size while keeping the necessary resolution to sample reasonably well the magnetic field structure on a spatial scale ${ }^{2}$ range from $\Lambda_{\min }=6$ up to $\Lambda_{\max } \simeq 770 \mathrm{kpc}$. Here we adopted the maximum range of spatial scales permitted by the computational grid. Observations reveal RM fluctuations on scales as low as $10 \mathrm{kpc}$ supporting this choice for $\Lambda_{\min }$. The exact value of $\Lambda_{\max }$ is more uncertain, however the presence in some cluster of galaxies of wide radio halos may indicate that at least in these cases the cluster magnetic field is spread over Mpc distances. Simulations with a different choice of of $\Lambda_{\max }$ are presented in Sect. 4.3.

The resulting two-dimensional power spectra of the RM images are shown in the top-left panel of Fig. 1. They have the same slope as their parent's three-dimensional magnetic field power spectra and they cover an equivalent range of spatial scales. However, it is evident from Fig. 1 that the same cluster magnetic field energy density will generate different magnetic field configurations with correspondingly different RM structures, for different values of the power spectrum spectral index.

\section{2. $R M$ radial profiles}

RM images of a whole cluster, such as those shown in Fig. 1, cannot be observed in real cases. However, it is relatively easy to measure the RM dispersion and mean $\left(\sigma_{\mathrm{RM}}\right.$ and $\left.\langle\mathrm{RM}\rangle\right)$ in limited regions by observing radio sources located at different projected distances from the center of a cluster. The $\sigma_{\mathrm{RM}}$ and $\langle\mathrm{RM}\rangle$ values found in radio sources, are usually calculated on their project surface which is typically about $50 \times 50 \mathrm{kpc}^{2}$ in size. Since we need to compare the simulation results with these observables, it is necessary to calculate the simulated values of $\sigma_{\mathrm{RM}}$ and $\langle\mathrm{RM}\rangle$ in regions with the same projected area.

Figure 2 shows the profiles of $\sigma_{\mathrm{RM}}$ (left column panels), $|\langle\mathrm{RM}\rangle|$ (central column panels) and $|\langle\mathrm{RM}\rangle| / \sigma_{\mathrm{RM}}$ (right column panels) as a function of the projected distance from the cluster center, calculated in boxes of $50 \times 50 \mathrm{kpc}^{2}$ in size. Due to the random nature of the simulated magnetic field, the values of $\sigma_{\mathrm{RM}}$ and $|\langle\mathrm{RM}\rangle|$ at a given radial distance vary from box to box. We calculated the mean and dispersion of $\sigma_{\mathrm{RM}}$ and $|\langle\mathrm{RM}\rangle|$ values from all the boxes located at the same projected distance from the cluster center. The lines and the shaded regions plotted in Fig. 2 represent the mean and dispersion of $\sigma_{\mathrm{RM}}$ and $|\langle\mathrm{RM}\rangle|$, respectively.

The size of the sampling region used to trace the RM profiles, falls inside the power spectrum range scales. Therefore, $\sigma_{\mathrm{RM}}$ is explained by the fact that the magnetic field fluctuates down to scales smaller than the size of the sampling regions.

\footnotetext{
${ }^{2}$ Here we refer to the length $\Lambda$ as to the magnetic field reversal scale. In this way, $\Lambda$ corresponds to a half-wavelength, i.e. $\Lambda=0.5$. $(2 \pi / k)$.
}

The most striking feature appearing in Fig. 2 is the significant amount of $\langle\mathrm{RM}\rangle$ predicted by the simulations for any of the three spectral indexes adopted. This is due to fluctuations of the cluster magnetic field on scales comparable or greater than the size of the sampling regions. This is not an unexpected result, but the numerical simulations provide a quantitative evaluation of this effect.

As expected, both $\sigma_{\mathrm{RM}}$ and $|\langle\mathrm{RM}\rangle|$ decrease from the cluster center outward, and the profiles corresponding to a constant magnetic field strength are the flattest. Moreover, the ratio of $|\langle\mathrm{RM}\rangle|$ to $\sigma_{\mathrm{RM}}$ as a function of distance from the cluster center is constant, i.e. both quantities have the same average radial gradient.

While both $\sigma_{\mathrm{RM}}$ and $|\langle\mathrm{RM}\rangle|$ increase linearly with the average cluster magnetic field strength, $\langle\boldsymbol{B}\rangle$, and the central gas density (see Eq. (2)), the ratio $|\langle\mathrm{RM}\rangle| / \sigma_{\mathrm{RM}}$ depends only on the magnetic field power spectrum slope, $n$, for a given range of fluctuation scales. This means that the comparison of RM data of radio galaxies embedded in a cluster of galaxies with simulated profiles, can be used to infer the strength and the power spectrum slope of the cluster magnetic field.

\subsection{The dependence of RM on the magnetic field largest scale structure}

To trace RM profiles shown in Fig. 2 we adopted the largest magnetic field fluctuation scale permitted by the computational grid size. In this section we consider different values for $\Lambda_{\max }$ and we calculate the corresponding variations of the simulated RM.

We performed an additional series of simulations where we decrease the value of $\Lambda_{\max }$ from $768 \mathrm{kpc}$ down to $6 \mathrm{kpc}$. We keep the minimum magnetic field scale $\Lambda_{\text {min }}$ fixed at $6 \mathrm{kpc}$ and allowed $\Lambda_{\max }$ to vary, since, given the limited size of the radio source RM images, the former can be better estimated from the data with respect to the latter.

The variation of $\sigma_{\mathrm{RM}},|\langle\mathrm{RM}\rangle|$ and their ratio as a function of $\Lambda_{\max }$ is shown in left, middle and right panel of Fig. 3, respectively.

Note that $\sigma_{\mathrm{RM}}$ and $|\langle\mathrm{RM}\rangle|$ in middle and left panels of Fig. 3 have been normalized to their values at $\Lambda_{\max }=768 \mathrm{kpc}$. Doing this we obtained a scaling factor for the profiles shown in Fig. 2 which depends only on the power spectrum slope $n$ and on $\Lambda_{\max }$ for a fixed $\Lambda_{\min }$. We found that for $n=3$, and especially $n=4$, $\sigma_{\mathrm{RM}}$ increases until $\Lambda_{\max }$ is larger than the size of the sampling region in which the statistic is calculated (i.e. $50 \times 50 \mathrm{kpc}^{2}$ ) while it stays almost constant for $n=2$. When $\Lambda_{\max }$ becomes smaller than the sampling region, $\sigma_{\mathrm{RM}}$ starts to decrease. Instead, $|\langle\mathrm{RM}\rangle|$ monotonically decrease by decreasing $\Lambda_{\max }$ and it goes to zero as $\Lambda_{\max } \rightarrow \Lambda_{\min }$ for all the three spectral indexes.

The right most panel of Fig. 3 shows the behavior of $|\langle\mathrm{RM}\rangle| / \sigma_{\mathrm{RM}}$ as a function of $\Lambda_{\max }$ for the three different slopes of the magnetic field power spectrum. In general we find the ratio $|\langle\mathrm{RM}\rangle| / \sigma_{\mathrm{RM}}$ decreases with $\Lambda_{\max }$.

If $\Lambda_{\max }$ is significantly larger than about $50-100 \mathrm{kpc}$, a given value of $|\langle\mathrm{RM}\rangle| / \sigma_{\mathrm{RM}}$ can be explained by different 

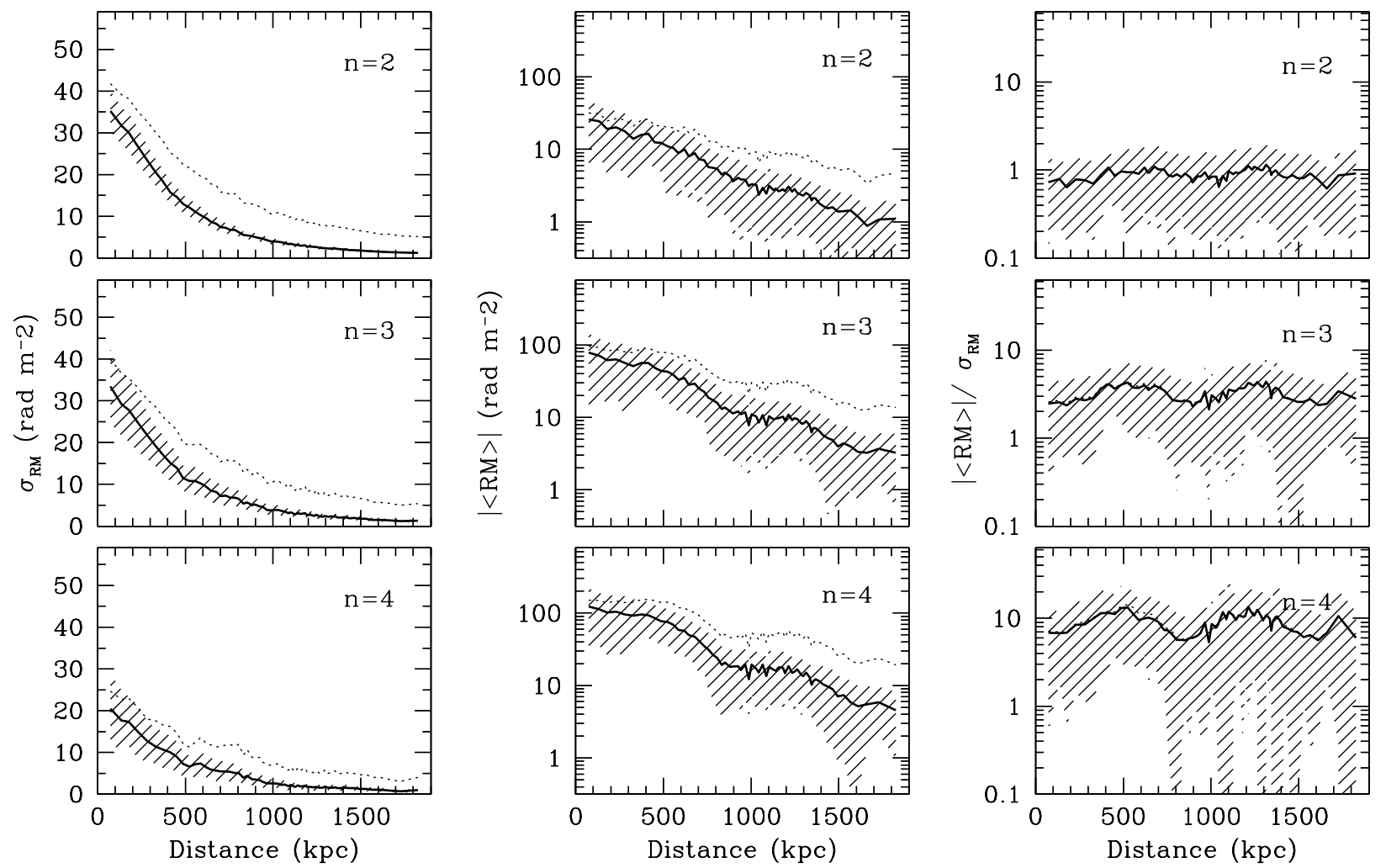

Fig. 2. Radial profiles obtained from the RM simulations described in Sect. 4. Left, central and right panels show the radial trends of $\sigma_{\mathrm{RM}}$, $|\langle\mathrm{RM}\rangle|$ and $|\langle\mathrm{RM}\rangle| / \sigma_{\mathrm{RM}}$, respectively, for three values of the index of the power spectrum $n$. Solid lines correspond to an average field at the cluster center of $\langle\boldsymbol{B}\rangle_{0}=1 \mu \mathrm{G}$ whose energy density decreases from the cluster center according to $B^{2} \propto n_{\mathrm{e}}(r)$, where $n_{\mathrm{e}}(r)$ is the gas density profile. The shaded regions indicate the 1-rms statistical fluctuations due to the random nature of the magnetic field. Dotted lines represent the profiles corresponding to a constant magnetic field strength of $\langle\boldsymbol{B}\rangle_{0}=1 \mu \mathrm{G}$ all over the cluster. Values have been sampled in $50 \times 50 \mathrm{kpc}{ }^{2}$ regions.
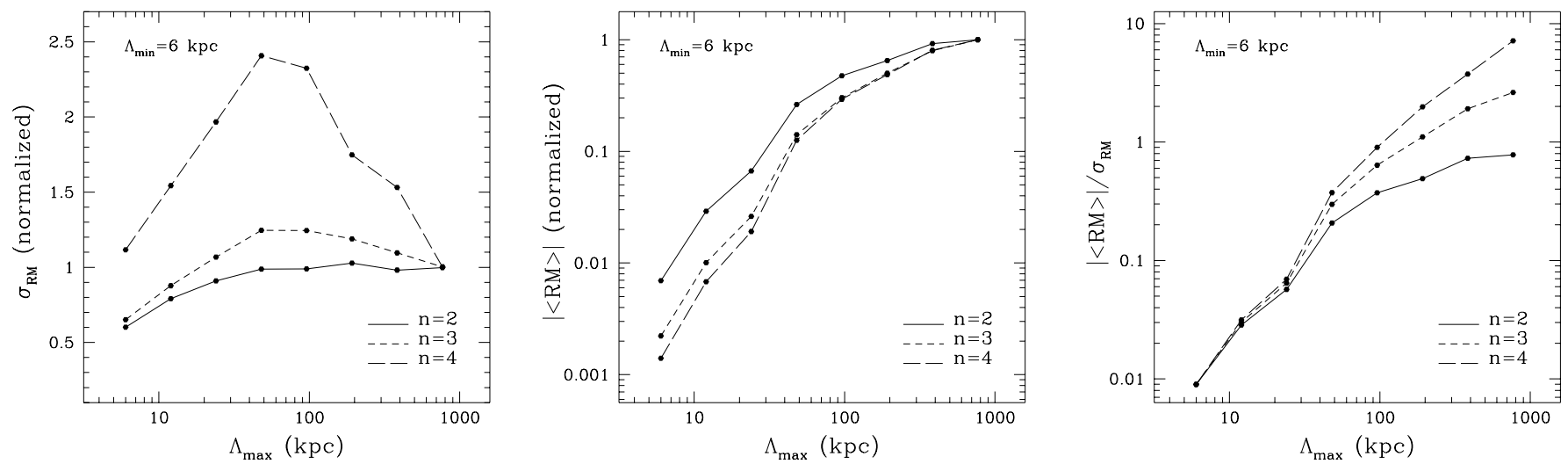

Fig. 3. Normalized dependence of $\sigma_{\mathrm{RM}}($ left $)$ and $|\langle\mathrm{RM}\rangle|($ middle $)$ as a function of $\Lambda_{\max }$, for the three spectral indexes $n=2,3$ and 4 . The right panel shows the ratio $|\langle\mathrm{RM}\rangle| / \sigma_{\mathrm{RM}}$ as a function of $\Lambda_{\max }$. The minimum magnetic field scale has been fixed at $\Lambda_{\min }=6 \mathrm{kpc}$ while $\sigma_{\mathrm{RM}}$ and $|\langle\mathrm{RM}\rangle|$ values have been sampled in $50 \times 50 \mathrm{kpc}^{2}$ regions.

combinations of parameters: the higher $n$, the lower $\Lambda_{\max }$. This could be considered as a serious degeneracy at a first glance. However, it is important to bear in mind that the resulting RM images, albeit characterized by the same value of $|\langle\mathrm{RM}\rangle| / \sigma_{\mathrm{RM}}$, still have very different spatial power spectra and thus different correlation lengths. Therefore, if good RM images are available for the cluster radio sources, it is possible to break the degeneracy between $n$ and $\Lambda_{\max }$, as will be shown for the case of A119 in Sect. 6.1.
As $\Lambda_{\max }$ becomes lower than about $50-100 \mathrm{kpc}$, the ratio $|\langle\mathrm{RM}\rangle| / \sigma_{\mathrm{RM}}$ falls below $0.2-0.3$ and its dependence on the power spectrum slope vanishes. In this case all the three spectral indexes predict almost the same $|\langle\mathrm{RM}\rangle| / \sigma_{\mathrm{RM}}$ which can be then used to constrain $\Lambda_{\max }$.

In any case, observed values of $|\langle\mathrm{RM}\rangle| / \sigma_{\mathrm{RM}}$ greater than unity can only be explained by steep magnetic field power spectra. For example, values of $|\langle\mathrm{RM}\rangle| / \sigma_{\mathrm{RM}}>1$ rule out $n=2$ for any value of $\Lambda_{\max }$. 


\subsection{Comparison with single-scale analytical magnetic field model}

The bulk of cluster magnetic field strength estimates appearing in the literature have been calculated so far by using an analytical formulation based on the approximation that the magnetic field is tangled on a single scale $\Lambda_{c}$. In this formulation, by considering a density distribution which follows a $\beta$-profile, the following relation (hereafter analytical formula) for the RM dispersion with the projected distance from the cluster center, $r_{\perp}$, is obtained by integrating Eq. (4):

$\sigma_{\mathrm{RM}}\left(r_{\perp}\right)=\frac{K B n_{0} r_{\mathrm{c}}^{1 / 2} \Lambda_{\mathrm{c}}^{1 / 2}}{\left(1+r_{\perp}^{2} / r_{\mathrm{c}}^{2}\right)^{(6 \beta-1) / 4}} \sqrt{\frac{\Gamma(3 \beta-0.5)}{\Gamma(3 \beta)}}$

where $\Gamma$ is the gamma function. The constant $K$ depends on the integration path over the gas density distribution: $K=624$, if the source lies completely beyond the cluster, and $K=441$ if the source is halfway the cluster. If the magnetic field scales with the distance from the cluster center as in Eq. (11), $\beta$ and $B$ in Eq. (15) must be replaced by $\beta+\mu$ and $B_{0}$, respectively (see Dolag et al. 2001).

It is of interest to compare the results of our simulations with the predictions of the analytical approach. However, since in this work we consider a magnetic field which fluctuates over a range of scales extending at least one order of magnitude, it is necessary to discuss which is the value for $\Lambda_{c}$ we should adopt in Eq. (15).

Here we consider three possibilities: i) $\Lambda_{c}$ is equal to the minimum field scale-length $\Lambda_{\text {min }}$; ii) $\Lambda_{\mathrm{c}}$ is equal to the field correlation length ${ }^{3}$ scale $\Lambda_{B_{z}}$ and iii) $\Lambda_{\mathrm{c}}$ is equal to the RM correlation length scale $\Lambda_{\mathrm{RM}}$.

In Fig. 4 we compare the $\sigma_{\mathrm{RM}}$ trend expected from the FARADAY simulations (solid line) with the predictions of the analytical formulation for the three scale lengths (dashed and dotted lines). The simulated trend corresponds to a magnetic field power spectrum with $n=2, \Lambda_{\text {min }}=6 \mathrm{kpc}$ and $\Lambda_{\max }=768 \mathrm{kpc}$. This model has a magnetic field correlation length of $\Lambda_{B_{z}} \simeq 16 \mathrm{kpc}$ and a RM correlation length of $\Lambda_{\mathrm{RM}} \simeq 69 \mathrm{kpc}$.

High-resolution RM maps of extended radio galaxies in clusters permit to detect patchy structures on small scales with typical size in the range $2-15 \mathrm{kpc}$ (e.g., Feretti et al. 1995, 1999a; Govoni et al. 2001b; Taylor et al. 2001; Eilek \& Owen 2002). The cluster magnetic fields strength reported in these works has been usually calculated by considering $\Lambda_{c}$

3 The magnetic field correlation length is defined as

$\Lambda_{B_{z}}=\frac{\int_{0}^{\infty} w_{z}(r) \mathrm{d} r}{w_{z}(0)}$

where $w_{z}(r)$ is the spherically averaged autocorrelation function of the magnetic field component along the line-of-sight. The RM correlation length is defined as

$\Lambda_{\mathrm{RM}}=\frac{\int_{0}^{\infty} C_{\mathrm{RM}}(r) \mathrm{d} r}{C_{\mathrm{RM}}(0)}$

where $C_{\mathrm{RM}}(r)$ is the radially averaged RM autocorrelation function.

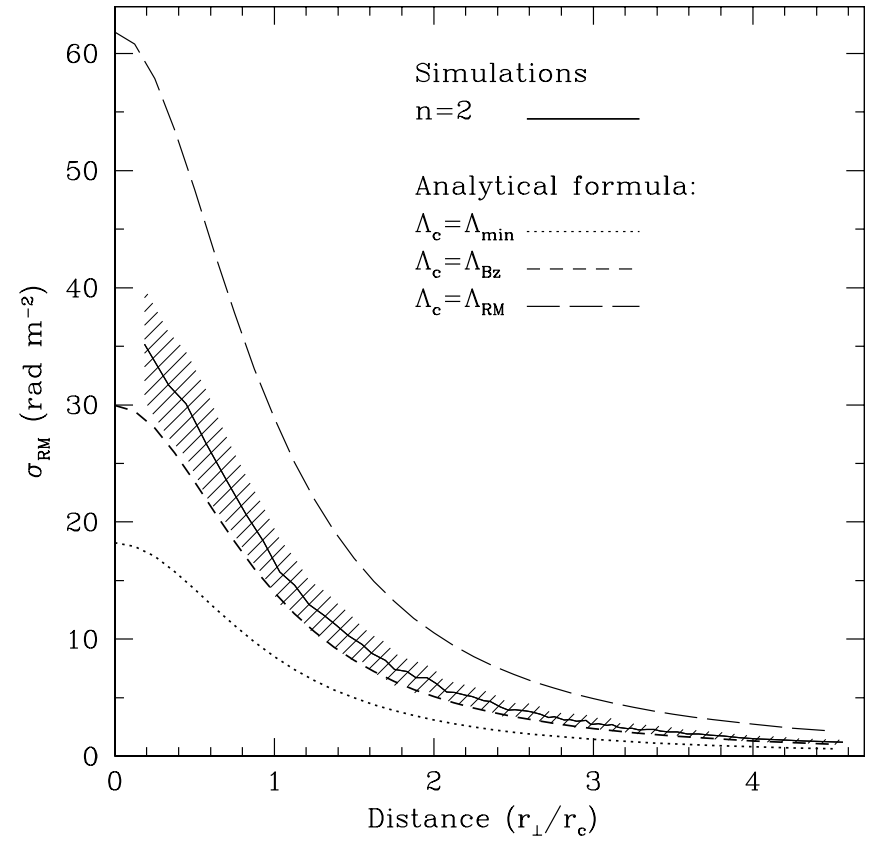

Fig. 4. Comparison of the $\sigma_{\text {RM }}$ profiles obtained from our simulations (solid line) and the analytical approximation (dashed and dotted lines). We assumed a source situated halfway through the cluster and the same magnetic field strength and gas density radial profiles in both the simulations and the analytical formulation. In the simulations we assumed a magnetic field power spectrum with a slope $n=2$ and a range of scales from $\Lambda_{\min }=6 \mathrm{kpc}$ to $\Lambda_{\max }=768 \mathrm{kpc}$.

in Eq. (15) to be equal to the smallest patchy structures detectable in the RM maps. This is the motivation for considering $\Lambda_{\mathrm{c}} \equiv \Lambda_{\text {min }}$. In this case, by assuming the same magnetic field strength, the single-scale magnetic field approximation leads to a lower $\sigma_{\mathrm{RM}}$ with respect to the prediction of our simulations. As a result the magnetic field strength inferred by the analytical formula is systematically overestimated by a factor of about 2 with respect the magnetic field obtained by assuming a multiscale magnetic field with a power spectral index of $n=2$.

On the other hand, since for a broad power spectrum $\Lambda_{\mathrm{RM}}>$ $\Lambda_{B_{z}}$ (see Vogt \& Enßlin 2003), the assumption of the RM correlation length for $\Lambda_{c}$ in Eq. (15) leads to underestimate the magnetic field strength by the same factor.

The best agreement between the simulated and analytical $\sigma_{\mathrm{RM}}$ trends is found for $\Lambda_{\mathrm{c}}=\Lambda_{B_{z}}$. In fact, this should be the proper scale length to use in Eq. (15).

To summarize, the analytical formulation leads to a reliable estimate for the average magnetic field strength provided that the magnetic field correlation length is known. Hoverer, since $\Lambda_{B_{z}}$ is $n$ dependent, we need to estimate the power spectrum of the magnetic field fluctuation in order to use Eq. (15).

\section{Simulated cluster radio sources depolarization}

In this section we investigate the effects of cluster magnetic fields on the depolarization of cluster radio sources. 

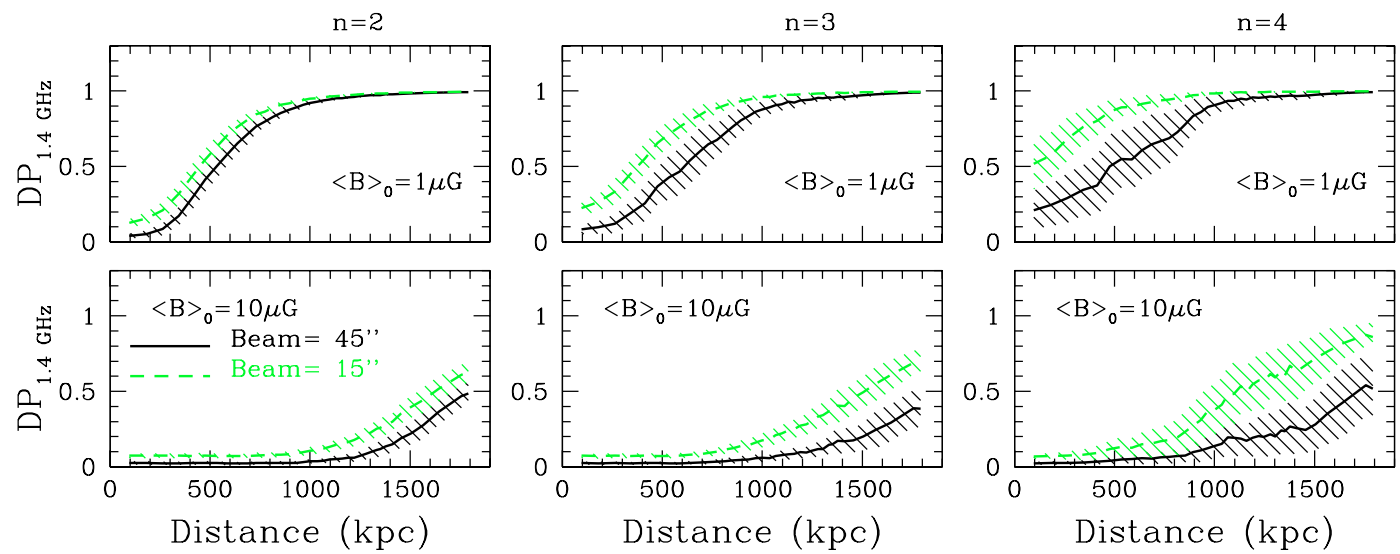

Fig. 5. Simulated 1.4 GHz beam-depolarization as a function of the distance from the cluster center in the case of a varying magnetic field $\left(\mu=0.3\right.$ ) with a central strength of $\langle\boldsymbol{B}\rangle_{0}=1$ (top panels) and $10 \mu \mathrm{G}$ (bottom panels). The simulated cluster is placed at a distance of about $300 \mathrm{Mpc}(z=0.05)$. Solid and dashed lines refer to a beam size of $45^{\prime \prime}$ and $15^{\prime \prime}$, respectively. At the distance of the cluster these beam widths correspond to projected linear sizes of about 60 and $20 \mathrm{kpc}$.

\subsection{Beam depolarization of cluster radio galaxies}

This kind of depolarization is expected to be particularly strong if differential Faraday rotation is occurring within the beam, i.e. if the minimum magnetic field scale length is smaller than the beam size.

Due to the high RM values in the inner regions of the cluster, the radio sources located in projection near the cluster center are expected to have a higher depolarization than those located at the periphery. In what follows we refer to the depolarization, DP, as the ratio between the polarization obtained after the rotation of the plane of polarization and the intrinsic polarization at a given frequency. To study the dependence of this effect on the cluster magnetic field strength and structure, we simulated the depolarization of virtual radio sources located halfway through the cluster at increasing distances from the cluster center. The virtual radio sources are supposed to lie at the distance of the cluster center and their three-dimensional structure and inclination are neglected. Their intrinsic polarization intensity, $P_{\text {Int }}$, was considered spatially uniform and completely ordered, i.e. the intrinsic polarization plane direction, $\Psi_{\text {Int }}$, did not vary across sources.

We first calculated the expected polarization plane direction, $\Psi_{\mathrm{Obs}}(v)$, at a given frequency along each line-of-sight of the sources according to Eq. (1).

We then converted $P_{\text {Int }}$ and $\Psi_{\mathrm{Obs}}$ into the Stokes parameters $Q(v)$ and $U(v)$, by inverting the relations:

$P=\sqrt{U^{2}+Q^{2}}$

$\Psi=\frac{1}{2} \arctan (U / Q)$.

Finally, the simulated $Q(v)$ and $U(v)$ images have been convolved with the desired beam and transformed back to $P_{\mathrm{Obs}}(v)$ and $\Psi_{\text {Obs }}(v)$ using Eqs. (18).

The parameters of the simulated cluster are those listed in Table 1. In Fig. 5 we show the depolarization of the sources at $1.4 \mathrm{GHz}$ as a function of the distance from the cluster center for two different decreasing ( $\mu=0.3$ ) magnetic field strength profiles with $\langle\boldsymbol{B}\rangle_{0}=1$ (top panels) and $10 \mu \mathrm{G}$ (bottom panels).
The simulated cluster is placed at a distance of about $300 \mathrm{Mpc}(z=0.05)$. Solid and dashed lines refer to a beam size of $45^{\prime \prime}$ and $15^{\prime \prime}$, respectively. At the distance of the cluster these beam widths correspond to projected linear sizes, $\Lambda_{\text {beam }}$, of about 60 and $20 \mathrm{kpc}$, i.e. $\Lambda_{\text {beam }} / \Lambda_{\text {min }} \simeq 10$ and $\Lambda_{\text {beam }} / \Lambda_{\text {min }} \simeq 3$, respectively.

We found that with a beam size of $45^{\prime \prime}$ the dependence of the simulated polarization profiles on the adopted power spectrum spectral index is only marginal, while it becomes significant by considering a beam of $15^{\prime \prime}$.

From Fig. 5 it is evident that the depolarization effect is strongly dependent on the cluster magnetic field strength. In the case of a weak magnetic field strength (e.g. $1 \mu \mathrm{G}$ ) the Faraday rotation only affects the polarization of the sources located in projection near the cluster center. As the average cluster magnetic field strength increases, the beam depolarization becomes significant at progressively larger distances. For a cluster magnetic field with a strength of $\langle\boldsymbol{B}\rangle_{0}=10 \mu \mathrm{G}$ in the center, the sources are severely depolarized for distances up to $2 \div 3 r_{\mathrm{c}}$ at both $45^{\prime \prime}$ and $15^{\prime \prime}$ resolutions.

The comparison of these simulations with the polarization properties of large samples of radio sources could provide an additional statistical constraint on the intracluster magnetic field strength.

\subsection{The morphology and (de)polarization of the radio halo emission}

Cluster radio halos may provide important information about the spatial power spectrum of the magnetic field fluctuations on large scales $(>100 \mathrm{kpc})$. In particular, different values of the power spectrum spectral index will generate very different total intensity and polarization brightness distributions for the radio halo emission.

For a given spectral index $n$, we calculated the expected total intensity and polarization synchrotron emission by introducing in the three-dimensional magnetic field an 
isotropic population of relativistic electrons whose distribution follows:

$N(\epsilon, \theta)=N_{0} \epsilon^{-\delta}(\sin \theta) / 2$

where $\epsilon$ and $\theta$ are the electron's energy and the pitch angle between the electron's velocity and the direction of the magnetic field, respectively.

At each point of the computation grid, we calculated the synchrotron emissivity, $J_{\text {syn }}$, by convolving the particle energy distribution given in Eq. (19) with the emission spectrum of the single electron

$-\frac{\mathrm{d} \epsilon}{\mathrm{d} t \mathrm{~d} v}=C_{\mathrm{f}} B \sin \theta F\left(\frac{v}{C_{v} B \sin \theta \epsilon^{2}}\right)$

where $F(x)$ is the synchrotron radiation kernel

$F(x)=x \int_{x}^{\infty} K_{5 / 3}(z) \mathrm{d} z$

where $C_{f}$ and $C_{v}$ are constants and whose numerical values (cgs units) are $6.26 \times 10^{18}$ and $2.34 \times 10^{-22}$, respectively, while $K_{5 / 3}$ is the $5 / 3$-order modified Bessel function.

Let $B_{\perp}$ be the magnetic field perpendicular to the line-ofsight. Because of the high beaming of the synchrotron radiation, only those electrons with $\theta=\sin ^{-1}\left(B_{\perp} / B\right)$ are visible by the observer. Therefore we have

$J_{\text {syn }}(v)=C_{\mathrm{f}} \frac{N_{0}}{2} \frac{B_{\perp}^{2}}{B} \int_{\epsilon_{\min }}^{\epsilon_{\max }} F\left(\frac{v}{C_{v} B_{\perp} \epsilon^{2}}\right) \epsilon^{-\delta} \mathrm{d} \epsilon$,

where $\epsilon_{\min }$ and $\epsilon_{\max }$ are respectively the low and high energy cut-offs of the electron distribution. We calculated the intrinsic linear polarization emissivity, $P_{\text {int }}$, according to

$P_{\mathrm{int}}(v)=J_{\mathrm{syn}}(v) \cdot \frac{\int_{\frac{\epsilon_{\min }}{\epsilon_{\max }}} F\left(\frac{v}{C_{v} B_{\perp} \epsilon^{2}}\right) \epsilon^{-\delta} \mathrm{d} \epsilon}{\int_{\epsilon_{\min }}^{\epsilon_{\max }} G\left(\frac{v}{C_{v} B_{\perp} \epsilon^{2}}\right) \epsilon^{-\delta} \mathrm{d} \epsilon}$

where $G(x)=x K_{2 / 3}(x)$ (see e.g., Rybicki \& Lightman 1979).

At our selected frequencies the radio halo is supposed to be optically thin, therefore we calculated the total intensity brightness distribution, $I_{\mathrm{syn}}(v)$, by simply integrating the emissivity in Eq. (22) along the line-of-sight

$I_{\text {syn }}(v)=\frac{1}{4 \pi} \int_{0}^{L} J_{\text {syn }}(v) \mathrm{d} l$

where $L$ is the computational grid size.

The calculation of the polarization brightness distribution must consider two important effects: i) since the direction of magnetic field changes from point to point inside the cluster also the orientation of the intrinsic polarization plane is a spatially varying quantity and ii) as the radio waves propagate through the magnetized intra-cluster medium their polarization plane is subject to the internal Faraday rotation. The well known result of this effect is a frequency dependent depolarization of the intrinsic synchrotron emission.

We first calculated the rotated polarization plane direction, $\Psi(v, l)$, of the emission from a volume element located at a position $l$ along the line-of-sight $(l=0$ correspond to the farthest side of the computational grid):

$\Psi(v, l)=\Psi_{\text {int }}(l)+(c / v)^{2} \times \operatorname{RM}(l)$

where $\Psi_{\text {int }}(l)$ is perpendicular to $B_{\perp}(l)$ and $\operatorname{RM}(l)$ is the internal Faraday rotation occurring in between the volume element and the observer:

$\operatorname{RM}(l)=812 \int_{l}^{L} n_{\mathrm{e}} B_{\|} \mathrm{d} l$.

It is then convenient again to convert $P_{\text {int }}(v)$ and $\Psi(v, l)$ given in Eqs. (23) and (25) into the Stokes parameters $Q(v, l)$ and $U(v, l)$, by inverting the relations given by Eqs. (18).

Finally, we obtained the simulated images of $Q(v)$ and $U(v)$ by adding together the contributes of all volumes along a given line-of-sight

$Q(v)=\int_{0}^{L} Q(v, l) \mathrm{d} l$
$U(v)=\int_{0}^{L} U(v, l) \mathrm{d} l$.

These simulated polarization images take into account of both the effects of the field ordering and internal Faraday rotation. Determining the origin and evolution of the relativistic electron population responsible for the halo emission in cluster of galaxy is beyond the purposes of this work. Here we restrict our interest to the study the dependence of the radio halo morphology and polarization on the magnetic field strength and structure. We adopted the simplest relativistic electrons distribution as possible which gives plausible values for the halo emission spectral index and total luminosity.

In the simulation we adopt an electron energy spectral in$\operatorname{dex} \delta=3$. We choose a Lorentz factor corresponding to the high-energy cutoff of the electron distribution, $\gamma_{\max }=$ $\epsilon_{\max } /\left(m_{\mathrm{e}} c^{2}\right)$, in a way that the high-frequency cutoff in the emission spectrum (at the cluster center) occurs at a frequency of $5 \mathrm{GHz}$ :

$\gamma_{\max }=3.4 \times 10^{4}\langle B\rangle_{0 \mu \mathrm{G}}^{-0.5} v_{5 \mathrm{GHz}}^{0.5}$.

The low-energy cutoff, $\gamma_{\min }$, and the relativistic electron density, $N_{0}$, are adjusted to guarantee a typical average halo brightness at $1.4 \mathrm{GHz}$ of $I_{1.4} \simeq 5 \times 10^{24} \mathrm{~W} \mathrm{~Hz}^{-1} \mathrm{Mpc}^{-2}$ (e.g., Giovannini et al. 1999) and a perfect equipartition between the relativistic electron and the magnetic field energy densities:

$\gamma_{\min } \simeq 1.9 \times 10^{3}\langle B\rangle_{0 \mu \mathrm{G}}^{-2}$.

The relativistic electron density $N_{0}$ follows the magnetic field energy density decrease so that the equipartition is satisfied at each distance from the cluster center.

We simulated the expected total intensity and polarization brightness distribution at $1.4 \mathrm{GHz}$ and $327 \mathrm{MHz}$, for three 
values of the magnetic field strength, namely $\langle B\rangle_{0}=0.5,1$ and $5 \mu \mathrm{G}$.

Due to the assumed declining $(\mu=0.3)$ mean magnetic field strength, the spectral index systematically increases from $\alpha_{1.4}^{327 \mathrm{GHz}} \simeq 1$ up to $\alpha_{1.4}^{327 \mathrm{GHz}} \simeq 1.3$ moving from the cluster center outward.

So far, polarization emission from radio halos has not been detected. The current upper limits to the polarization at $1.4 \mathrm{GHz}$ are a few percent (3-4\%) for beams of about $45^{\prime \prime}$. Due to the low spatial resolution of these observations, the beam depolarization effect is expected to be very strong. Therefore, we simulated the polarization intensity expected at $45^{\prime \prime}$ resolution for a radio halo located at a redshift $z=0.05$. At this distance, the corresponding linear resolution is of about $60 \mathrm{kpc}$. In the left-column panels of Fig. 6 we show the simulated radio halo brightness and polarization percentage distributions at $1.4 \mathrm{GHz}$ for three different values of the power spectrum spectral index $n$. In the right-column panels of Fig. 6 we show the expected fractional polarization profiles at $1.4 \mathrm{GHz}$ and $327 \mathrm{MHz}$ for the different values of the average magnetic field strength.

As one would expect, we found that the degree of polarization percentage:

i) increases with increasing values of $n$;

ii) increases with the frequency $v$;

iii) decreases towards the cluster center;

iv) decreases with increasing magnetic field strength.

Our results indicate that a power spectrum slope steeper than $n=3$ and a magnetic field strength lower than $\sim 1 \mu \mathrm{G}$ result in a radio halo polarization percentage at a frequency of $1.4 \mathrm{GHz}$ that is far in excess the current observational upper limits at $45^{\prime \prime}$ resolution. This means that either the power spectrum spectral index is flatter than $n=3$ or the magnetic field strength is significantly higher than $\sim 1 \mu \mathrm{G}$. The halo depolarization at $327 \mathrm{MHz}$ is particularly severe and the expected polarization percentage at this frequency is always below $1 \%$.

We also found that the magnetic field power spectrum slope has a significant effect in shaping the radio halo. In particular, flat power spectrum indexes $(n<3)$ give raise to very smooth radio brightness images (under the assumption that the radiating electrons are uniformly distributed). RM images of radio galaxies in conjunction with observations of the halo brightness and polarization distributions could provide information on the behavior of the non-thermal (magnetic field and relativistic electrons) components of the intra-cluster medium over a large range of spatial scales. For this reason, it is important to apply our approach to those cluster with both types of radio sources, such as A2255, in order to determine if a single magnetic field power spectrum can account for both the observed RM and radio halo images (Govoni et al. 2002; Govoni et al. 2004, in preparation).

\section{Application to the data}

\subsection{The case of Abell 119}

The cluster of galaxy A119 is an ideal study case to apply our new approach. It is characterized by three extended radio galaxies, 0053-015, 0053-016 and 3C 29, located at different projected distances from the cluster center, respectively at 170, 453 and $1515 \mathrm{kpc}$ (see Table 2 and Fig. 10a). All these sources are highly polarized and they have been studied in detail with the Very Large Array (VLA) by Feretti et al. (1999a). From RM images with a resolution of $3.75^{\prime \prime}(\simeq 4.4 \mathrm{kpc})$ Feretti et al. (1999a) estimated a tangling scale of $\sim 10 \mathrm{kpc}$ and, by using the simple analytical approach (see Sect. 4.4), they inferred a strength of about $5 \mu \mathrm{G}$ for an assumed constant intra-cluster magnetic field. However, Dolag et al. (2001) by comparing the X-ray surface brightness with the $\sigma_{\mathrm{RM}}$ of the three radio galaxies (see Sect. 3.1) estimated that the magnetic field strength in A119 scales as $B \propto n_{\mathrm{e}}^{0.9}$. Taking into account such a varying magnetic field, the analytical approach yields a central magnetic field of $\sim 7.5 \mu \mathrm{G}$.

We used FARADAY in order to numerically obtain the expected RM images for the radio galaxies of Abell 119. In the numerical simulations we used the gas density distribution deduced by X-ray observations for this cluster $\left(r_{\mathrm{c}}=378 \mathrm{kpc}\right.$, $n_{0}=1.18 \times 10^{-3} \mathrm{~cm}^{-3}$ and $\beta=0.56$; Cirimele et al. 1997), and we left the magnetic field power spectrum slope and strength as free parameters. All three radio galaxies are assumed to lie on a plane perpendicular to the line-of-sight at the distance of cluster center. The details of the three-dimensional structure of the radio source are neglected, and the entire observed Faraday rotation is assumed to occur in the cluster intergalactic medium.

A119 is located in a region of low Galactic RM (see Table 2) and therefore we apply no correction to the observed $\langle\mathrm{RM}\rangle$ with this respect. We compared both $\sigma_{\mathrm{RM}}$ and $|\langle\mathrm{RM}\rangle|$ versus the X-ray surface brightness $S_{\mathrm{x}}$ taken from Dolag et al. (2001) to estimate the index $\mu$ for the radial scaling of the magnetic field. Figure 7 shows the same dependence RM $\propto S_{x}^{1.1}$ for both quantities, in agreement with the result by Dolag et al. (2001). From Eq. (14), with $\beta=0.56$ and $\alpha=1.1$, it follows that $\mu \simeq 0.5$.

As a first step we compared the observed $\sigma_{\mathrm{RM}}$ and $|\langle\mathrm{RM}\rangle|$ with the expectation of the simulations. Since the linear resolutions of both the computational grid and the observations are very similar, we do not apply any Gaussian smoothing to the simulated RM images. Figure 8 show the best fit of the simulated $\sigma_{\mathrm{RM}}$ radial profiles for the radio galaxies in A119. The typical uncertainties in the observed RMs are of the order of $10-15 \mathrm{rad} \mathrm{m}^{-2}$. However, a realistic error budget should also consider the effects of a number of systematic uncertainties which are difficult to estimate, such as the relative distance of the galaxies from the cluster center along the line-of-sight and the statistical fluctuations which are intrinsic to the random nature of the magnetic field. Moreover, the "observed" RMs themselves are the result of a fit procedure as outlined in Sect. 2. For all these reasons, we decided to use a simplified approach, i.e., a non-weighted least square fit without attempting to include either $\sigma_{\mathrm{RM}}$ nor $\langle\mathrm{RM}\rangle$ uncertainties in the fit procedure.

In the fit procedure we fixed $n$ at a given value and let the magnetic field strength as a free parameter. The best fit of the magnetic field strength, obtained by fitting the $\sigma_{\mathrm{RM}}$ profiles (Fig. 8, left), gives $\langle\boldsymbol{B}\rangle_{0} \simeq 5 \mu \mathrm{G}$ for each $n$. This is the mean magnetic field at the cluster center. Since the modeled 
$10 \quad \stackrel{20}{30}$

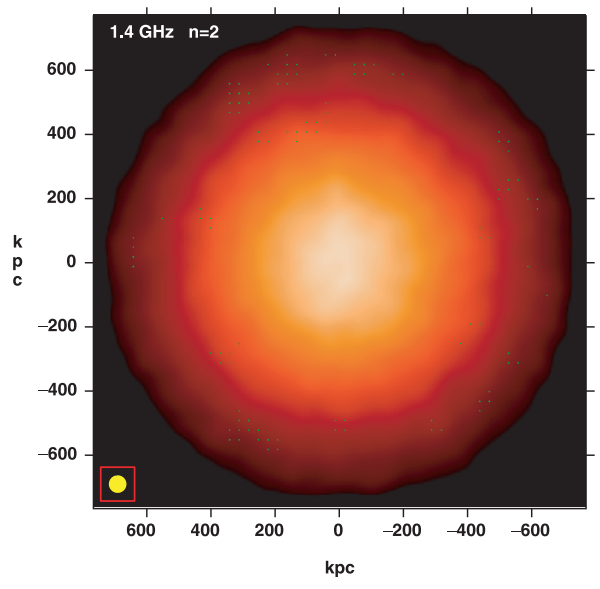

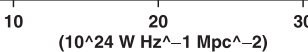

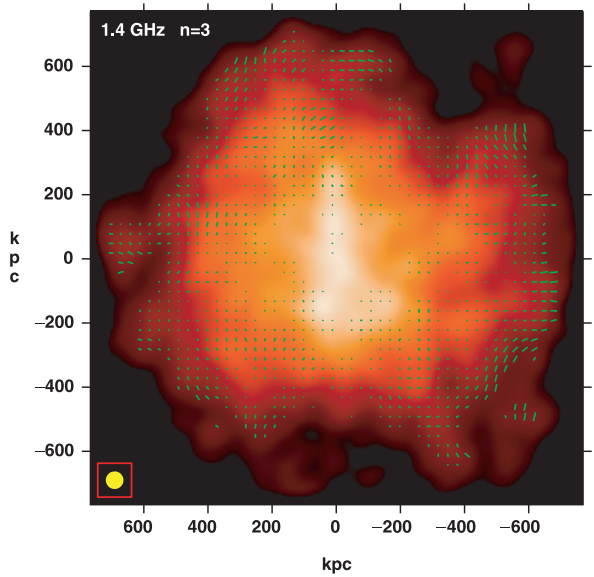

(10)

$10 \underbrace{20}_{\left(10^{\wedge} 24 \mathrm{~W} \mathrm{~Hz}^{\wedge}-1 \mathrm{Mpc}^{\wedge}-2\right)}$

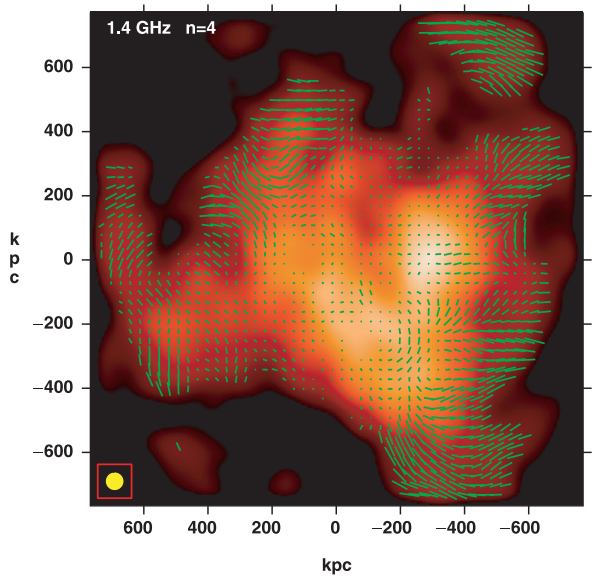

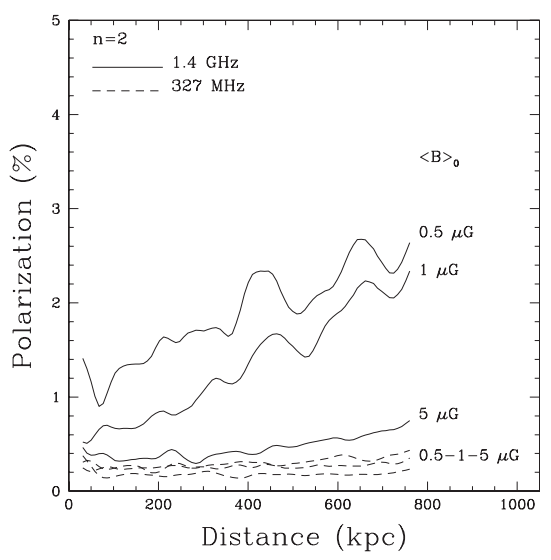
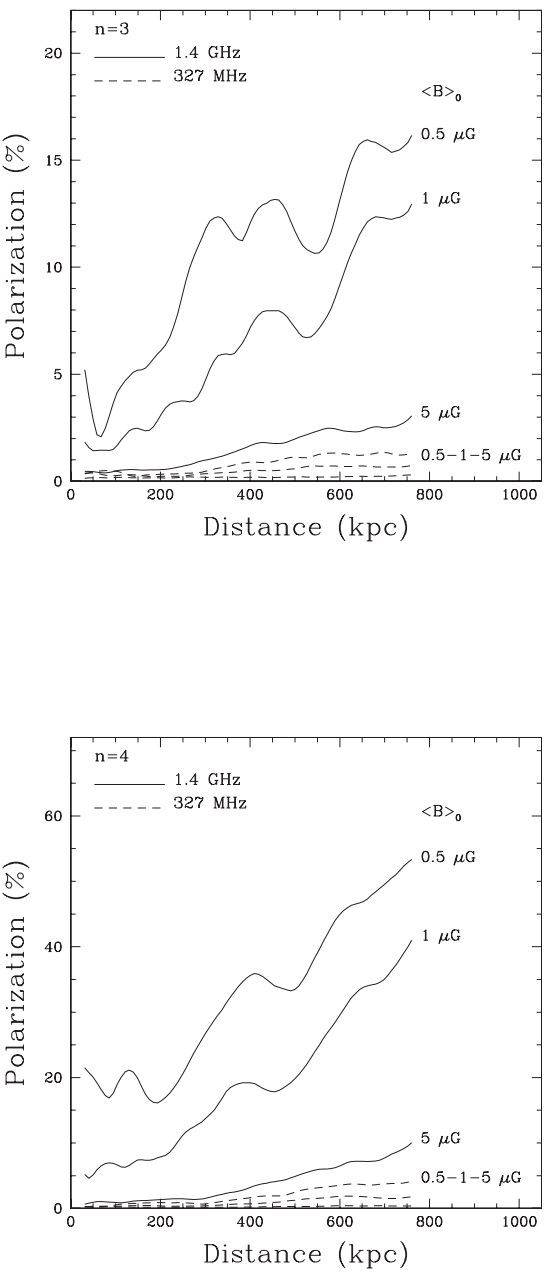

Fig. 6. Simulated synchrotron halo emission for a cluster at a distance of $z=0.05$ as it would be observed with a beam of $45^{\prime \prime}(\simeq 60 \mathrm{kpc}$ ). Left panels: simulated halo brightness and polarization images at $1.4 \mathrm{GHz}$ for different values of the magnetic field power spectrum slope $n$ and $\langle B\rangle_{0}=1 \mu \mathrm{G}$; the vectors lengths are proportional to the degree of polarization, with 100 percent corresponding to $100 \mathrm{kpc}$ on the sky. Field directions are those of the E-vector. Right panels: radially averaged profiles of the polarization percentage at $327 \mathrm{MHz}$ and $1.4 \mathrm{GHz}$ for three values of the magnetic field strength, namely $\langle B\rangle_{0}=0.5,1$ and $5 \mu \mathrm{G}$. 
magnetic field strength decreases from the cluster center outward $(\mu=0.5)$, the mean field strength within a spherical volume of radius $r=3 r_{\mathrm{c}}$ (with $r_{\mathrm{c}}=378 \mathrm{kpc}$ ) gives $\langle\boldsymbol{B}\rangle \simeq 1.5 \mu \mathrm{G}$. The comparison between simulated and observed $|\langle\mathrm{RM}\rangle|$ (Fig. 8, right) allows us to constrain the value of the index of the power spectrum to $n \simeq 2$. Fits corresponding to models with $n>2$ are definitely poorer as evident from the high $|\langle\mathrm{RM}\rangle|$ values they predict. Also models characterized by $n<2$ are a poorer fit because their low expected $|\langle\mathrm{RM}\rangle|$ values are incompatible with the observations. As shown in bottom panel of Fig. 8, the Kolmogorov spectral index $(n=11 / 3)$ gives a reasonable fit to the data only if the maximum scale of the magnetic field fluctuations is about $100 \mathrm{kpc}$.

As expected from the discussion in Sect. 4.3, the observed $\sigma_{\mathrm{RM}}$ and the $|\langle\mathrm{RM}\rangle|$ profiles, can be explained either by a broad and flat $(n=2)$ or by a narrower but steeper $(n=11 / 3$, $\Lambda_{\max }=100 \mathrm{kpc}$ ) magnetic field power spectrum.

A way to discriminate the two models is to compare the structure functions of the observed and simulated RM images of the individual radio galaxies. As an example, in Fig. 9 we show the observed and simulated RM images relative to the source nearest to cluster center: 0053-015. The model with $n=2$ is in remarkable agreement with the observed RM features. This is confirmed by the structure functions ${ }^{4}$ plotted in the bottom left panel of Fig. 9. The observed RM structure function cuts off below a linear scale of about $10 \mathrm{kpc}$. This cutoff and the overall shape of the observed RM structure functions is reproduced well by the model with $n=2$. We note that even if the magnetic field power spectrum with the Kolmogorov index $n=11 / 3$ and $\Lambda_{\max }=100$ fits the observed RM profiles, it generates a structure function which is not in agreement with the data. We found similar results for the other two sources (not shown).

\subsubsection{The composite RM image}

To confirm the above results we calculated the two-dimensional power spectrum of a composite RM image of A119 obtained by combining all together the data of the three radio galaxies of the cluster (Fig. 10a). In Fig. 10b we shown the power spectrum of the "mask", i.e. a window function image which is equal to unity if the line-of-sight intercepts one of the sources and zero otherwise. The power spectrum of the composite RM image is shown in Fig. 10c. This is the convolution of the true power spectrum with the power spectrum of the mask. Since the power spectrum of the mask is quite broad, we cannot measure the power spectrum slope directly from the image shown in Fig. 10c. However, we can multiply by the same mask the simulated RM image corresponding to the models with $n=2$

\footnotetext{
${ }^{4}$ To calculate the structure function of the RM images we first construct the structure function RM matrix

$S(\mathrm{~d} x, \mathrm{~d} y)=\left\langle[\operatorname{RM}(x, y)-\operatorname{RM}(x+\mathrm{d} x, y+\mathrm{d} y)]^{2}\right\rangle$

where $\mathrm{d} x$ and $\mathrm{d} y$ are both positive and negative offsets and the ensemble average is calculated over all the positions, $(x, y)$, in the RM image. The RM structure function is then obtained by radially averaging $S(\mathrm{~d} x, \mathrm{~d} y)$ in annuli of increasing size $\Lambda=\sqrt{\mathrm{d} x^{2}+\mathrm{d} y^{2}}$.
}
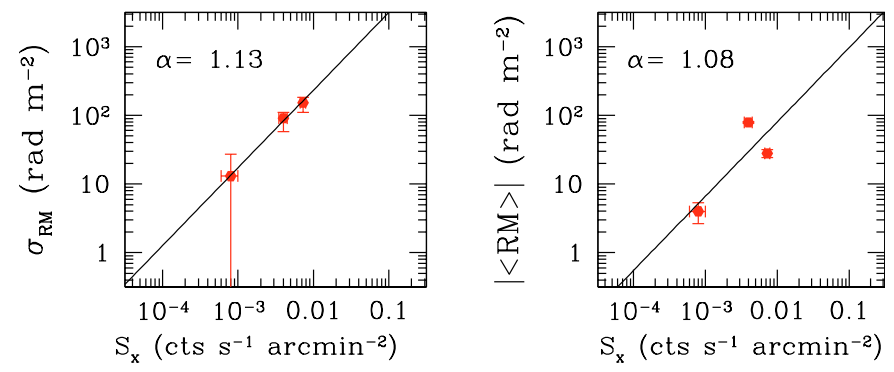

Fig. 7. Correlation of the RM with the X-ray surface brightness for the three radio galaxies in A119. We found $\mathrm{RM} \propto S_{\mathrm{x}}^{1.1}$.

and $n=11 / 3$ with $\Lambda_{\max }=100$, calculate their power spectra, and compare them with the observed one. The radially averaged profiles of the observed (solid line) and simulated (dashed lines) power spectra of the composite RM image are shown in Fig. 10d. Again, we find that the model with $n=2$ gives the best description of the data.

To summarize, the best fit of both the RM profiles, structure functions and power spectra observed for the three extended radio galaxies in A119 is obtained for a spectral index $n=2$, $\Lambda_{\text {min }}=6 \mathrm{kpc}, \Lambda_{\max }=770 \mathrm{kpc}$ and $\langle\boldsymbol{B}\rangle_{0} \simeq 5 \mu \mathrm{G}$. The mean field strength within a spherical volume of radius $r=3 r_{\mathrm{c}}$ (with $r_{\mathrm{c}}=378 \mathrm{kpc}$ ) gives $\langle\boldsymbol{B}\rangle \simeq 1.5 \mu \mathrm{G}$. The observed $|\langle\mathrm{RM}\rangle| / \sigma_{\mathrm{RM}}$ ratios rule out $n=1$ while models with $n>2$, compatible with the constraint $\Lambda_{\max } \simeq 100 \mathrm{kpc}$, produce RM images whose structure functions and power spectra are incompatible with the data. These results are based on the assumption that the observed Faraday rotation is produced entirely by a magnetized intra-cluster medium.

\subsection{Variations of the power spectrum spectral index among clusters}

We have seen in the case of A119 how by comparing the simulated RMs with the observed RMs obtained for radio sources located at different projected distances from the cluster center, it is possible to gain important information about the magnetic field power spectrum and strength. However, one important question concerns the variation of the magnetic field power spectrum among clusters of galaxies. To explore this issue, we collected a sample of nine clusters and groups of galaxies (without cooling flows) for a total of 14 radio sources for which high-quality RM data are available in the literature. The cluster sample we chose for this study is listed in Table 2. Since we are interested in the mean and dispersion of the RM, we included in this analysis only those clusters hosting radio sources for which high-resolution RM images are available.

The clusters in our sample are characterized by quite different gas density profiles, linear size and probably magnetic field strengths. However, since these parameters affect both $|\langle\mathrm{RM}\rangle|$ and $\sigma_{\mathrm{RM}}$ in the same way, it is possible to combine the $\mathrm{RM}$ data from different clusters and use the ratio $|\langle\mathrm{RM}\rangle| / \sigma_{\mathrm{RM}}$ to study the variation of the spectral index $n$ among them from a statistical point of view.

The mean of the RM is a fundamental parameter for our analysis and therefore the contribution of our own Galaxy to 
Table 2. RM data taken from the literature.

\begin{tabular}{lcccccc}
\hline \hline Cluster & Source & $\begin{array}{c}\text { Distance } \\
(\mathrm{kpc})\end{array}$ & $\begin{array}{c}\text { Gal. RM } \\
\left(\mathrm{rad} / \mathrm{m}^{2}\right)\end{array}$ & $\begin{array}{c}\langle\mathrm{RM}\rangle \\
\left(\mathrm{rad} / \mathrm{m}^{2}\right)\end{array}$ & $\sigma_{\mathrm{RM}}$ & Ref. \\
\hline Coma & NGC 4869 & 192 & -6 & -127 & 181 & 1 \\
\hline A119 & $0053-015$ & 170 & -1 & +28 & 152 & 2 \\
& $0053-016$ & 453 & & -79 & 91 & \\
& 3C 29 & 1515 & & +4 & 13 & \\
\hline 3C 129 & 3C 129.1 & 50 & -10 & +21 & 200 & 3 \\
& 3C 129 & 500 & & -125 & 82 & \\
\hline A514 & PKS0405-203 & 2098 & -3 & -19 & 48 & 4 \\
& PKS0446-206 & 750 & & 46 & 47 & \\
\hline A2255 & B1712+640 & 392 & -6 & -91 & 78 & 5 \\
& B1713+641 & 612 & & +67 & 59 & \\
\hline Zw0056.9+2636 & NGC 326 E-lobe & 70 & -36 & -16 & 58 & 6 \\
& NGC 326 W-lobe & 35 & & -21 & 60 & \\
\hline A400 & 3C 75 & 0 & 4 & -7.6 & 100 & 7 \\
\hline A2634 & 3C 465 & 0 & -60 & -25 & 120 & 7 \\
\hline 3C 449 & 3C 449 & 0 & -165 & -166 & 38 & 8 \\
\hline
\end{tabular}

Column 1: Cluster name; 2: Source name; 3: Source projected distance from cluster center; 4: Galactic RM contribute; 5: RM mean; 6: RM dispersion; 7: References.

References: 1) Feretti et al. (1995); 2) Feretti et al. (1999a); 3) Taylor et al. (2001); 4) Govoni et al. (2001a); 5) Govoni et al. (2003) in preparation; 6) Murgia et al. (2001); 7) Eilek \& Owen (2002); 8) Feretti et al. (1999b).

this quantity should be removed carefully. We determined the galactic contribution to the $\langle\mathrm{RM}\rangle$ for each cluster of our sample according to the values reported by Simard-Normandin et al. (1981). We use the distance-weighted mean of the RMs of all sources located within \pm 15 deg of galactic longitude and latitude, respectively. The galactic RM contributions are listed in Table 2 (Col. 4).

Figure 11 shows the trend of the observed $|\langle\mathrm{RM}\rangle| / \sigma_{\mathrm{RM}}$ ratios as a function of the sampling region size $\left(\Lambda_{\mathrm{s}}\right)$ for $n=1$, 2,3 and 4 . The observed values of the $|\langle\mathrm{RM}\rangle| / \sigma_{\mathrm{RM}}$ ratio, lie above 0.1 and below 2 for all clusters except for 3C 449, which has a very low $|\langle R M\rangle|$ if corrected for the galactic RM contribution. It is important to note that $3 \mathrm{C} 449$ belongs to a small group and is located at low galactic latitude, therefore the correction for the galactic RM contribution should be considered carefully since it could strongly influence the result. The mean of $|\langle\mathrm{RM}\rangle| / \sigma_{\mathrm{RM}}$ ratio calculated for the 14 radio sources is $\simeq 0.6$.

The values of $|\langle\mathrm{RM}\rangle| / \sigma_{\mathrm{RM}}$ expected from the simulations for $n>3$ exceed those observed by a significant amount. Indeed, as already found for A119, the rather flat spectral index $n \simeq 1-2$ represents the best description of the data. The relatively large dispersion of the observed data is largely consistent with what expected from the intrinsic statistical scatter shown in the right panel of Fig. 2 (the scatter is larger towards the lower values of the $|\langle\mathrm{RM}\rangle| / \sigma_{\mathrm{RM}}$ ratio).
In A2255, for both radio galaxies the mean of the observed $\mathrm{RM}$ is greater than its dispersion: $|\langle\mathrm{RM}\rangle| / \sigma_{\mathrm{RM}} \simeq 1.2$. This is a strong indication that, at least for this cluster, the magnetic field power spectrum might be steeper than in the other cases. The inferred power spectrum index could be as high as $n=2$ or more. A2255 is a very interesting cluster since it also contains a wide synchrotron radio halo and the magnetic field power spectrum slope has a major role in determining the large scale morphology of such a diffuse emission (see Sect. 5.2).

\section{Summary}

In this paper we present a numerical approach to investigate the strength and structure of magnetic fields permeating the intergalactic medium in clusters of galaxies. The estimates of cluster magnetic field strengths appearing in the literature have been calculated through their effects on the polarization properties of radio galaxies in or behind clusters by using an analytical formulation based on the approximation that the magnetic field is tangled on a single scale. However, detailed observations of these radio sources and MHD simulations suggest that it is necessary to consider cluster magnetic fields which fluctuate over a wide range of spatial scales. To accomplish this we simulated a random three-dimensional magnetic field with a power law power spectrum in a cubical box. We calculated the effects of the expected Faraday rotation on the polarization 

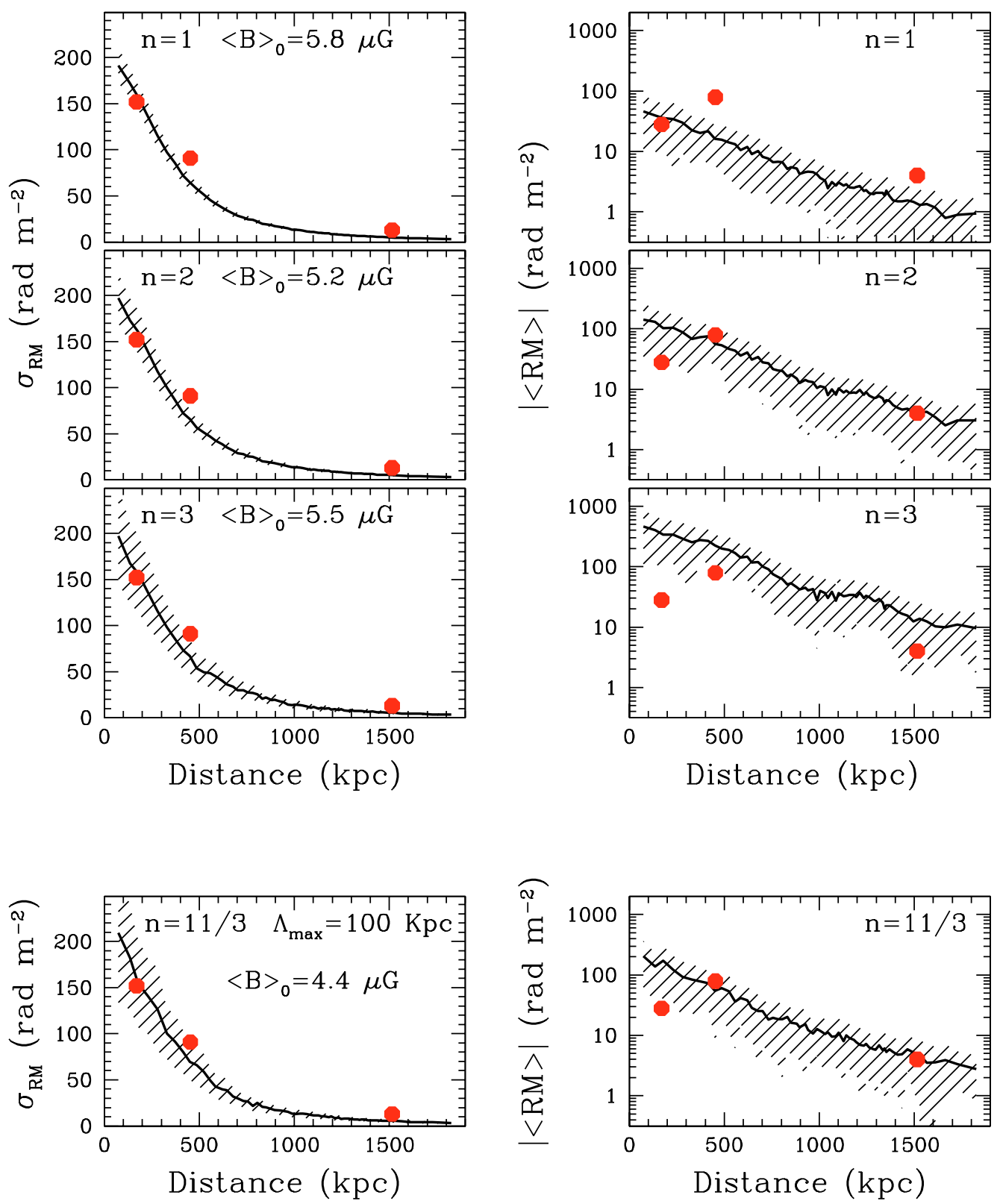

Fig. 8. Best fit of the simulated RM $\sigma_{\mathrm{RM}}$ profiles for the cluster A119 (left column panels). The comparison of simulated and observed $|\langle\mathrm{RM}\rangle|$ is shown in right column panels. Solid dots refer to the observed values for the radio galaxies 0053-015, 0053-016 and 3C 29. The best fit to the central magnetic field strength gives $\langle\boldsymbol{B}\rangle_{0} \simeq 5 \mu \mathrm{G}$. Since the modeled magnetic field strength decreases from the cluster center outward $\left(\mu \simeq 0.5\right.$ ), the mean field strength within a spherical volume with radius $r=3 r_{\mathrm{c}}$ is $\langle\boldsymbol{B}\rangle \simeq 1.5 \mu \mathrm{G}$. We find that the spectral index $n=2$ gives the best description of the data if the magnetic field power spectrum ranges from a minimum scale of $6 \mathrm{kpc}$ up to a maximum scale of $768 \mathrm{kpc}$. The fit corresponding to the models with $n \leq 1$ and $n \geq 2$ are definitely poorer since to the $|\langle\mathrm{RM}\rangle| / \sigma_{\mathrm{RM}}$ ratios they predict are too low and too high, respectively. The Kolmogorov spectral index $(n=11 / 3)$ gives a reasonable fit to the data only if the maximum scale of the magnetic field fluctuations is about $100 \mathrm{kpc}$ (bottom panels).

properties of radio cluster sources such as radio galaxies and radio halos whose radiation crosses the magnetized intracluster medium. The main issues of this paper are summarized as follows:

- We determined the statistical behavior of the RM over a typical radio galaxy-sized region as a function of distance from the cluster center for different values of the magnetic field power spectrum slope and range of scales. The $|\langle\mathrm{RM}\rangle| / \sigma_{\mathrm{RM}}$ ratio is an easily measurable observable that is expected to reflect the relative amplitude of large to smallscale magnetic field fluctuations. The numerical simulations allow a quantitative evaluation of this effect. We found that the $|\langle\mathrm{RM}\rangle| / \sigma_{\mathrm{RM}}$ ratio depends rather critically on the power spectrum slope, $n$, and therefore it can be used to constrain this parameter if spatially-resolved RM images of radio sources located at different distances from the cluster center are available.

- The magnetic field strength estimated by our simulations in the case of $n=2$ (i.e. most of the magnetic field energy 

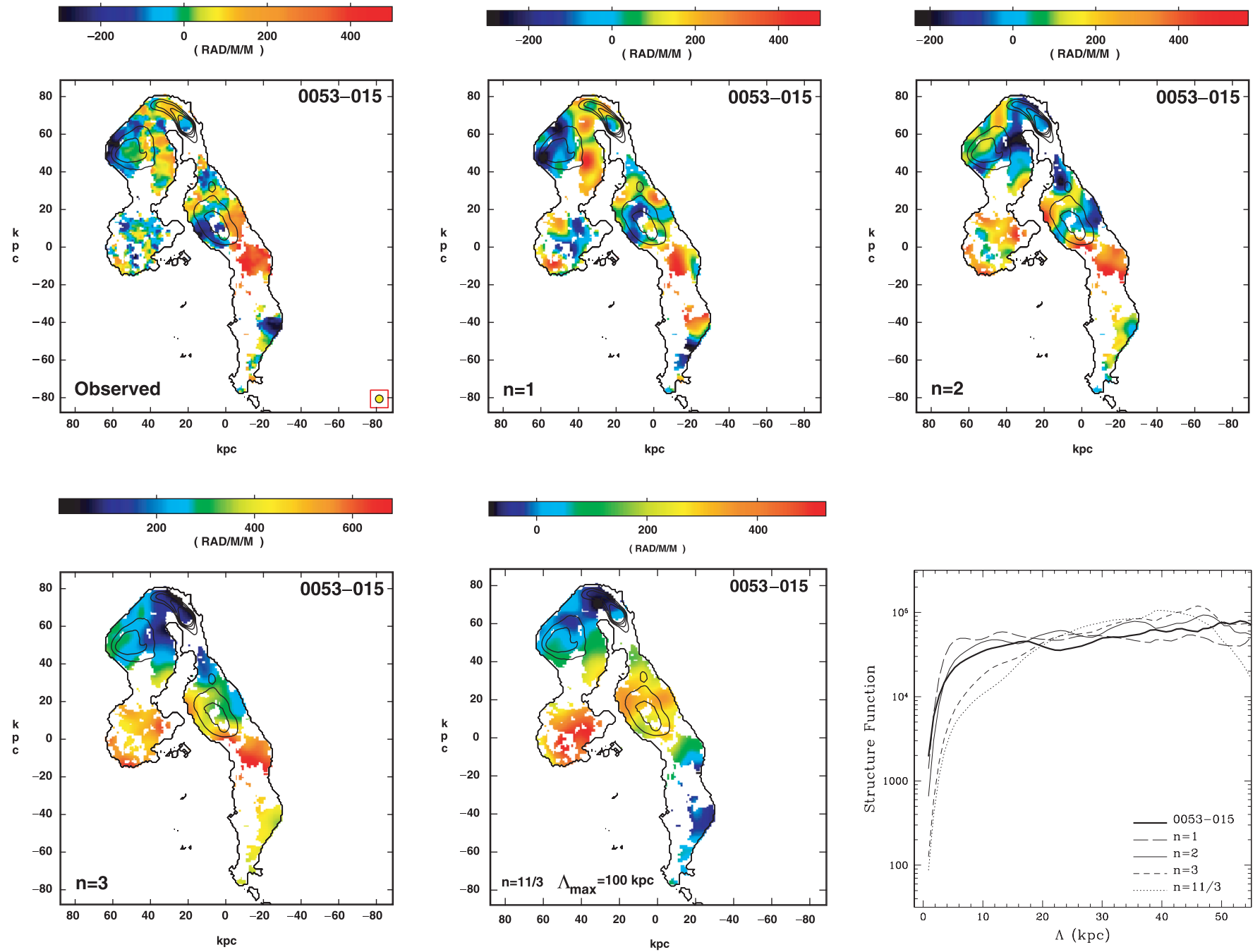

Fig. 9. Comparison of observed and simulated RM images of the source 0053-016 in A119. The observed RM image is shown in the top left panel. Models with $n \simeq 2$ better reproduce the observed RM features. This is confirmed by the structure functions of the observed and simulated RM images shown in the bottom left panel.

resides in the small scales) results in a factor of about 2 lower strengths than the expectations based on the singlescale magnetic field approximation (see Fig. 4). The analytic formulation leads to a reliable estimate for the average magnetic field strength only provided that the magnetic field correlation length is adopted (i.e. the magnetic field power spectrum is known).

- We simulated the expected dependence of the statistical beam depolarization of marginally resolved radio sources located at increasing distances from the cluster center. We found that the amount of depolarization is strongly dependent on the magnetic field strength.

- We point out that radio halos may provide important information about the spatial power spectrum of the magnetic field fluctuations on large scales. In particular, different values of the power spectrum spectral index give rise to very different total intensity and polarization brightness distributions. So far, polarization emission from radio halos have not been detected. Our results indicate that a power spectrum slope steeper than $n=3$ and a magnetic field strength lower than $\sim 1 \mu \mathrm{G}$ predict a radio halo polarization percentage at a frequency of $1.4 \mathrm{GHz}$ that is far in excess the current observational upper limits at 45" resolution. This means that either the power spectrum spectral index is flatter than $n=3$ or the magnetic field strength is significantly higher than $\sim 1 \mu \mathrm{G}$ or both.

- In the last part of the paper, simulations and data have been compared through an interactive approach. The cluster A119 is an ideal case for our new approach. It is characterized by three extended radio galaxies located at different projected distances from the cluster center and it is located in a region of very low Galactic RM. The best fit of both the RM profiles and image structure functions observed for the three extended radio galaxies in A119 is obtained for a spectral index $n=2$ and $\langle\boldsymbol{B}\rangle_{0} \simeq 5 \mu \mathrm{G}$. This is the mean magnetic field at the cluster center, the mean field strength within a spherical volume with radius $r=3 r_{\mathrm{c}}$ is $\langle\boldsymbol{B}\rangle \simeq 1.5 \mu \mathrm{G}$. The observed $|\langle\mathrm{RM}\rangle| / \sigma_{\mathrm{RM}}$ ratios rule out $n=1$ while models with $n>2$, 

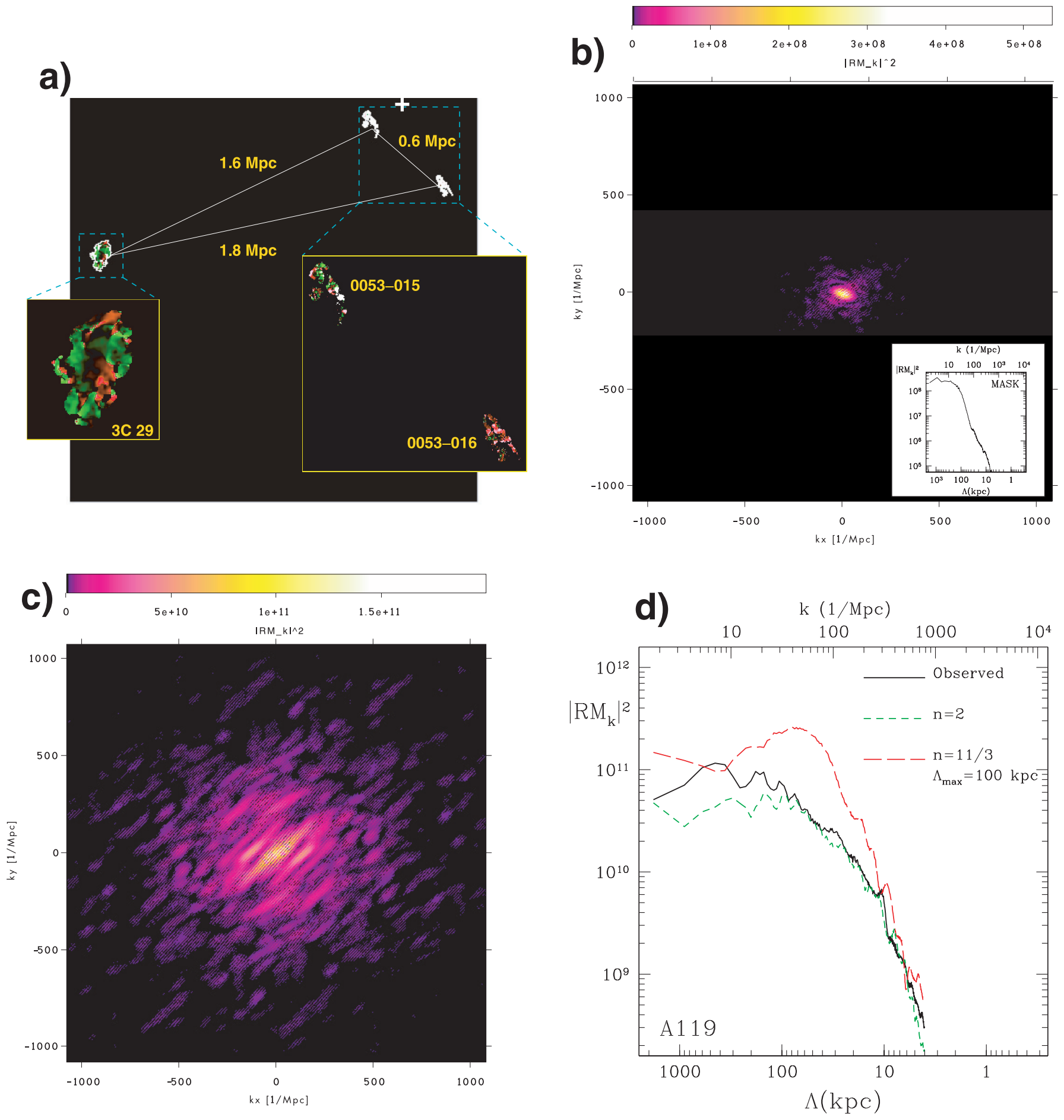

Fig. 10. a) Composite RM image of A119 obtained by combining together the individual images of the three radio galaxies. The cross marks the position of the X-ray centroid. b) The power spectrum of the mask window function. The short-wavenumber modes are due to the interference between the source-to-source baselines. The inset shows the radially averaged profile of the mask power spectrum. c) Power spectrum of the composite RM image. d) Comparison of the observed (solid line) and simulated (dashed lines) radially averaged RM power spectra, see text.

compatible with the constraint $\Lambda_{\max } \simeq 100 \mathrm{kpc}$, produce $\mathrm{RM}$ images whose power spectra are incompatible with the data.

- An as-yet unexplored issue concerns the variation of the magnetic field power spectrum among cluster of galaxies. For this purpose, we collected a sample of nine clusters (without cooling flows) for a total of 14 radio sources for which high-quality RM data are available in literature.
The values of $|\langle\mathrm{RM}\rangle| / \sigma_{\mathrm{RM}}$ expected from the simulations for $n>3$ exceed the observed ones by a significant amount. Indeed, as already found for A119, the rather flat spectral index $n \simeq 2$ represents the best description of the data.

Acknowledgements. The authors thank the referee, Dr. De Young, for suggestions that have improved the paper. We are indebted to 


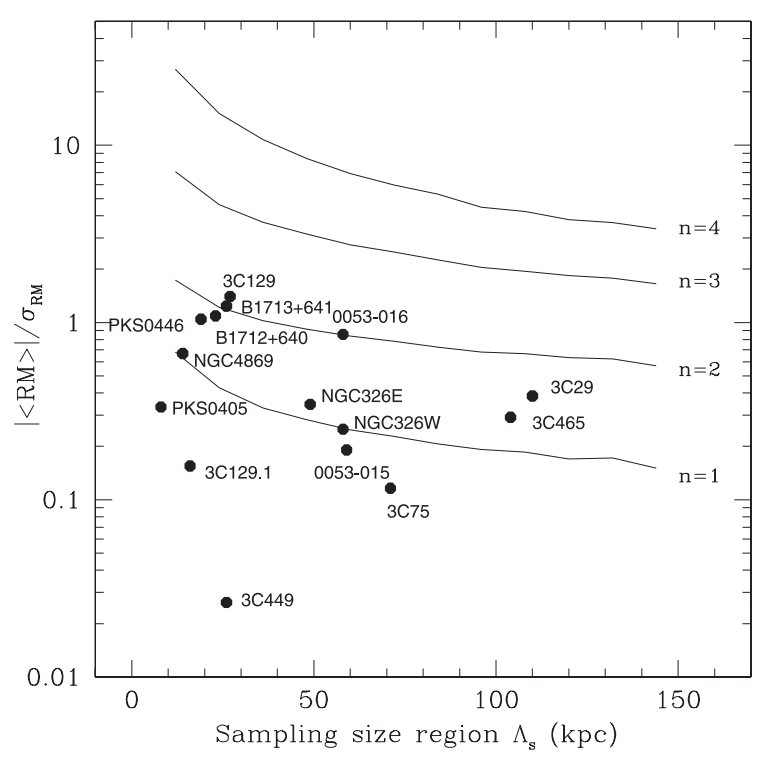

Fig. 11. The ratio $|\langle\mathrm{RM}\rangle| / \sigma_{\mathrm{RM}}$ as a function of the sampling region. The horizontal lines represent the simulated trends corresponding to $n=1,2,3$ and 4 .

Hans-Rainer Kloeckner who provided us the galactic RM tables. We also thank Gianfranco Brunetti for stimulating discussions and useful comments. K. Dolag acknowledge support by a Marie Curie Fellowship of the European Community program "Human Potential" under contract number MCFI-2001-01227.

\section{References}

Allen, S. W., Taylor, G. B., Nulsen, P. E. J., et al. 2001, MNRAS, 324, 842

Bacchi, M., Feretti, L., Giovannini, G., \& Govoni, F. 2003, A\&A, 400, 465

Brunetti, G. 2002, Matter and energy in clusters of galaxies, ed. S. Bowyer, \& C.-Y. Hwang [arXiv: astro-ph/0208074]

Carilli, C. L., \& Taylor, G. B. 2002, ARA\&A, 40, 319

Cirimele, G., Nesci, R., \& Trevese, D. 1997, ApJ, 475, 11

Clarke, T. E., Kronberg, P. P., \& Böhringer, H. 2001, ApJ, 547, L111

Dolag, K., Schindler, S., Govoni, F., \& Feretti, L. 2001, A\&A, 378, 777

Dolag, K., Bartelmann, M., \& Lesch, H. 2002, A\&A, 387, 383

Eilek, J. A., \& Owen, F. N. 2002, ApJ, 567, 2002
Enßlin, T. A., \& Vogt, C. 2003, A\&A, 401, 835

Felten J. E. 1996, in Clusters, lensing, and the future of the Universe, ed. V. Trimble, \& A. Reisenegger, ASP Conf. Ser., 88, 271

Feretti, L., Dallacasa, D., Giovannini, G., \& Tagliani, A. 1995, A\&A, 302,680

Feretti, L., \& Giovannini, G. 1996, in Extragalactic radio sources, ed. R. Ekers, C. Fanti, \& L. Padrielli (Kluwer Academic, Publisher), IAU Symp., 175, 333

Feretti, L., Dallacasa, D., Govoni, F., et al. 1999a, A\&A, 344, 472

Feretti, L., Perley, R., Giovannini, G., \& Andernach, H. 1999b, A\&A, 341, 29

Fusco-Femiano, R., dal Fiume, D., Feretti, L., et al. 1999, ApJ, 513, L21

Fusco-Femiano, R., Orlandini, M., Brunetti, G., et al. 2004, ApJ, 602, L73

Giovannini, G., Feretti, L., Venturi, T., Kim, K.-T., \& Kronberg, P. P. 1993, ApJ, 406, 399

Giovannini, G., Tordi, M., \& Feretti, L. 1999, New Astron., 4, 141

Govoni, F., Feretti, L., Giovannini, G., et al. 2001a, A\&A, 376, 803

Govoni, F., Taylor, G. B., Dallacasa, D., Feretti, L., \& Giovannini, G. 2001b, A\&A, 379, 807

Govoni, F., Enßlin, T. A., Feretti, L., \& Giovannini, G. 2001c, A\&A, 369,441

Govoni, F., Feretti, L., Murgia, M., et al. 2002, in Matter and energy in Clusters of galaxies, ed. S. Bowyer, C.-Y. Hwang, \& Chung-Li, Taiwan [arXiv: astro-ph/0211292]

Lawler, J. M., \& Dennison, B. 1982, ApJ, 252, 81

Murgia, M., Parma, P., de Ruiter, H. R., et al. 2001, A\&A, 380, 102

Newman, W. I., Newman, A. L., \& Rephaeli, Y. 2002, ApJ, 575, 755

Perley, R. A., \& Taylor, G. B. 1991, AJ, 101, 1623

Press, W. H., Flannery, B. P., \& Teukolsky, S. A. 1986 (Cambridge: University Press), 1986

Rephaeli, Y., \& Gruber, D. 2002, ApJ, 579, 587

Rephaeli, Y., Gruber, D., \& Blanco, P. 1999, ApJ, 511, L21

Rybicki, G. B., \& Lightman, A. P. 1979 (New York: Wiley-Interscience), 1979

Rudnick, L., \& Blundell, K. M. 2003, ApJ, 588, 143

Simard-Normandin, M., Kronberg, P. P., \& Button, S. 1981, ApJS, 45, 97S

Taylor, G. B., \& Perley, R. A. 1993, ApJ, 416, 554

Taylor, G. B., Govoni, F., Allen, S., \& Fabian, A. C. 2001, MNRAS, 326,2

Tribble, P. C. 1991a, MNRAS, 250, 726

Tribble, P. C. 1991b, MNRAS, 253, 147

Vogt, C., \& Enßlin, T. A. 2003, A\&A, 412, 373 